\title{
Absorption spectrum of the quasar HS1603+3820
}

\section{Observations and data analysis ${ }^{\star}$}

\author{
A. Dobrzycki ${ }^{1}$, M. Nikolajuk ${ }^{2}$, J. Bechtold $^{3}$, H. Ebeling ${ }^{4}$, B. Czerny ${ }^{5}$, and A. Różańska ${ }^{5}$ \\ 1 ESO, Karl-Schwarzschild-Strasse 2, 85748 Garching bei München, Germany \\ e-mail: adam.dobrzycki@eso.org \\ 2 Faculty of Physics, University of Białystok, Lipowa 41, 15424 Białystok, Poland \\ e-mail:mrk@alpha.uwb.edu.pl \\ 3 Steward Observatory, University of Arizona, Tucson, AZ 85721, USA \\ e-mail: jbechtold@as.arizona.edu \\ 4 Institute for Astronomy, University of Hawai'i, 2680 Woodlawn Drive, Honolulu, HI 96822, USA \\ e-mail: ebeling@ifa.hawaii.edu \\ 5 Copernicus Astronomical Center, Bartycka 18, 00716 Warszawa, Poland \\ e-mail: bcz, agata@camk.edu.pl
}

Received 22 February 2007 / Accepted 27 September 2007

\section{ABSTRACT}

\begin{abstract}
Context. We present multi-wavelength observations of the bright quasar HS1603+3820: the optical data taken with the MMT and Keck telescopes, with the $40-50 \mathrm{~km} \mathrm{~s}^{-1}$ resolution, and X-ray data taken by the Chandra X-ray Observatory satellite.

Aims. The optical spectra contain a very large number of absorption lines from numerous heavy elements. Our goal is to analyze these features to obtain constraints on the properties of associated absorbers, to be used in modeling of the quasar intrinsic flux and properties of the clouds.

Methods. We have determined the properties - column densities and redshifts - of the individual components. We derived the X-ray properties of HS1603+3820 and the optical-to-X-ray slope index $\alpha_{\text {ox }}$.

Results. We found $\alpha_{\text {ox }}$ of 1.70 , which is at the high end of the typical range for a radio quiet quasar. We found 49 individual heavy element absorption clouds, which can be grouped into eleven distinct systems. Absorbers from the associated system, which likely is the one spatially closest to the quasar, show large C IV to H I column density ratio, reaching $\sim 20$.

Conclusions. Intrinsic X-ray properties of the quasar are typical. Determination of column densities of ions (including hydrogen) gives a strong foundation for modeling of the quasar ionising flux.
\end{abstract}

Key words. quasars: absorption lines - quasars: individual: HS1603+3820

\section{Introduction}

HS1603 +3820 is a $z_{\mathrm{em}}=2.54$ quasar discovered during the Hamburg/CfA Bright Quasar Survey (Dobrzycki et al. 1996). With $B=15.9$, it is among the top few brightest quasars known in the $z_{\mathrm{em}}=2-3$ redshift interval (Véron-Cetty \& Véron 2006). The most striking feature of HS1603+3820 is, however, the extreme richness of its heavy element absorption spectrum, and in particular, the $z_{\mathrm{abs}} \approx z_{\mathrm{em}}$ absorption.

The absorption spectrum of HS1603+3820 was first described in Dobrzycki et al. (1999, hereafter D99), based on medium-resolution (90-100 $\mathrm{km} \mathrm{s}^{-1}$ ) spectrum obtained in 1997 April with the MMT and the Blue Channel spectrograph. Although in this spectrum many of the absorption systems were blended, it was clear that the quasar held great promise for the analysis of the properties of associated absorbers.

^ Based on the observations collected at the MMT, which is a joint facility of the Smithsonian Institution and the University of Arizona. Some of the data presented herein were obtained at the W. M. Keck Observatory, which is operated as a scientific partnership among the California Institute of Technology, the University of California and the National Aeronautics and Space Administration. The Observatory was made possible by the generous financial support of the W. M. Keck Foundation.
The absorption systems that are spatially close to the quasar can be used as probes for the intrinsic emission of the quasar itself, since the conditions in the systems are undoubtedly heavily influenced by the quasar flux (e.g. Crenshaw et al. 2003; Gabel et al. 2005; Scott et al. 2005, and references therein). Systems that contain lines originating from various species and various ionisation levels are particularly interesting, since they can provide an independent way of determining physical properties of the absorbing medium, which can cast light at the intrinsic quasar continuum in the far UV band as well as the absorbing cloud formation.

With that as the principal goal, we initiated a project of multi-wavelength observations of HS1603+3820, aiming to obtain spectral information at various wavelengths. In this paper we present the results of our X-ray and optical observations of the quasar. In the forthcoming paper (Różańska et al. 2007, in preparation, Paper II), we use those observations to constrain models of the quasar broad band continuum and the cloud confinement.

In the meantime, Misawa et al. (2003, 2005, hereafter referred to as M03 and M05, respectively) observed HS1603+3820 in 2002 March and 2003 July in very high resolution $\left(6.7 \mathrm{~km} \mathrm{~s}^{-1}\right)$, using the Subaru telescope with the High Dispersion Spectrograph. The primary goal of these 
observations was to establish the nature of the absorbers (intervening or associated), using the analysis of line time variability, partial coverage and profile shapes. High resolution of their observations enabled resolving many of the systems into a number of narrow components. M03 and M05 grouped the absorbers into several systems, designated with the letters from A to $\mathrm{H}$. In our optical spectroscopy data we see all those systems and three additional intervening systems. For consistency, and to enable easy comparisons, wherever possible we will use these designations throughout this paper.

In a very recent paper, Misawa et al. (2007, hereafter M07) expanded the analysis of absorber variability, using new spectra from the Subaru and Hobby-Eberly telescopes, and confirmed the finding from M05 that C IV in System A is highly variable. This paper also described one system (labeled I) that they did not have in their older data. This system was seen in our data, and for consistency with M07 we rewrote parts of this paper to also use this designation.

Our optical spectra are of lower resolution ( $F W H M$ of $\left.40-50 \mathrm{~km} \mathrm{~s}^{-1}\right)$, but they complement the Subaru data in two important areas: they have higher signal-to-noise ratio (100-140 per pixel in the C IV and Ly $\alpha$ regions) and they cover much larger wavelength range $(\sim 3700$ to $\sim 10000 \AA)$. The former reduces uncertainty in blended regions containing thick lines and helps in finding weak lines, while the latter allows analyzing lines from species inaccessible in the Subaru spectra. The most important addition is detection and analysis of hydrogen lines, not seen in Misawa et al.'s spectra, as well as detection of heavy-element lines other than C IV in System A.

This paper is organised as follows. In Sect. 2 we discuss the Chandra X-ray observation of HS1603+3820. In Sect. 3 we present our optical observations of HS1603+3820, and we discuss the individual systems and compare our results with those of Misawa et al., discussing the possible sources of discrepancies. We comment on the absorber variability in Sect. 4. We summarize the results in Sect. 5.

\section{X-ray observations}

HS1603+3820 was observed for $9.2 \mathrm{ks}$ with the Chandra X-ray Observatory on 2002 November 29 (ObsID 4026), with the 128 row subarray of the ACIS-S3 chip in VFAINT mode, with frame time of $0.4 \mathrm{~s}$. The effective good time for the observation was $8.3 \mathrm{ks}$.

The data were reduced using the tools available in the CIAO v. 3.3 and SHERPA software packages ${ }^{1}$, using Chandra calibration database (CALDB) v. 3.2.1. Because of large calibration uncertainties for soft X-rays, the data with $E<0.5 \mathrm{keV}$ were excluded from the analysis. We extracted source and background spectra from the regions around the quasar using dmextract. In total, the net photon yield from the quasar was 145 photons, allowing for most fundamental spectral fits only. We note that the ACIS pile-up is not a problem, both because of the low source count rate and because of the low frame time resulting from the use of the chip subarray.

We performed spectral fits with SHERPA, applying the newest gain corrections to the event list. We performed the fit assuming the intrinsic quasar spectrum to be a power law, $A E^{-\gamma}$, with fixed Galactic absorption towards HS1603+3820 of $N_{\mathrm{H}}=1.3 \times 10^{21} \mathrm{~cm}^{-2}$, as obtained with COLDEN ${ }^{2}$. The best

\footnotetext{
${ }^{1}$ http://cxc.harvard.edu/ciao/

${ }^{2}$ http://cxc.harvard.edu/toolkit/colden.jsp
}

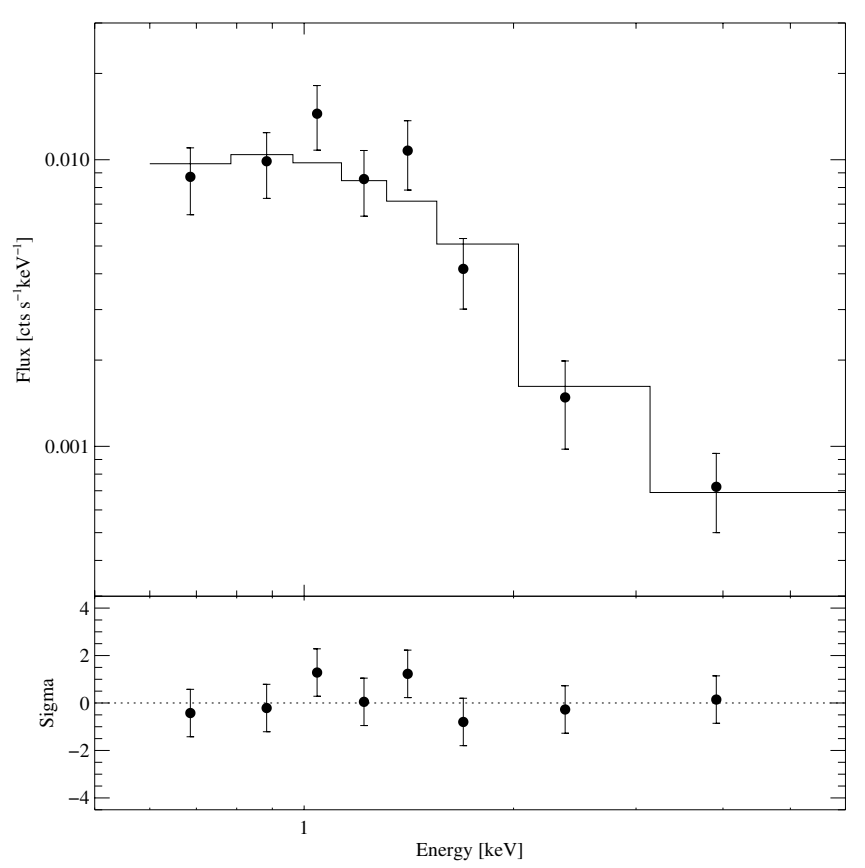

Fig. 1. Chandra ACIS-S3 X-ray spectrum of HS1603+3820 and best fit power law plus Galactic absorption. The bottom panels shows the fit residuals.

fit gave the photon spectral index of $\gamma=1.91 \pm 0.20$ and normalization at (rest) $1 \mathrm{keV}$ of $A=(2.43 \pm 0.79) \times 10^{-4}$ photons $\mathrm{cm}^{-2} \mathrm{~s}^{-1} \mathrm{keV}^{-1}$. The slope of the spectrum is within the typical range for quasars (e.g. Fiore et al. 1998; Reeves \& Turner 2000). The spectrum and the fit are shown in Fig. 1. We also attempted fits in which we allowed for additional absorption from the quasar, but there was no improvement in the fit.

The combination of the above result with the spectrophotometric data from Scott et al. (2000) yields the value of the optical-to-X-ray slope, $\alpha_{\mathrm{ox}}=$ $-\log \left(f_{v, 2 \mathrm{keV}} / f_{v, 2500 \mathrm{~A}}\right) / \log \left(v_{2} \mathrm{keV} / v_{2500 \mathrm{~A}}\right)$, of 1.70 , which is at the high end of the typical parameter range at $z_{\mathrm{em}} \approx 2.5$ (Bechtold et al. 2003).

\section{Optical spectroscopy}

HS1603+3820 has been observed by us with two instruments. On 2001 August 19 we used the ESI, Echelle Spectrograph and Imager (Sheinis et al. 2002), on the Keck II telescope, with the MIT-LL $2048 \times 4096$ CCD, with the $0.5^{\prime \prime}$ slit. Observing conditions were excellent throughout the night, with clear sky and seeing of the order of $0.7^{\prime \prime}$. The combined exposure time was 4800 s. The usable part of the spectrum covered the 23900 to $\sim 10000 \AA$ wavelength range and had the resolution of $45-50 \mathrm{~km} \mathrm{~s}^{-1}$, with the signal-to-noise ratio peaking at $\sim 140$ (per pixel) near the Ly $\alpha$ emission line, gradually falling to $\sim 100$ near the $\mathrm{C}$ IV emission line and then to $\sim 70$ in the red end.

On 2003 June 21 we used the Blue Channel Spectrograph on the MMT, in the echelette/cross-dispersed mode, using the Lesser $2688 \times 512$ "ccd35" detector with the 1 " slit. The observing conditions were variable. Seeing became worse at the end of the night, reaching $\sim 2$ arcsec, and the conditions were adversely affected by the moon age of $\sim 0.75$ and smoke from forest fires. The combined exposure time was $18000 \mathrm{~s}$. The usable spectrum ranged from $\sim 3750$ to $\sim 7100 \AA$ and had the resolution 
of $40-45 \mathrm{~km} \mathrm{~s}^{-1}$. The signal-to-noise ratio in this spectrum is in the 110-130 (per pixel) range throughout the entire wavelength range.

For both sets of observations, the initial reduction was done in the standard way with IRAF. We note that despite several attempts, the extracted MMT spectrum appears to show residual flux. Even lines that are clearly saturated, which in the Keck spectrum have black cores, do not reach zero intensity in their bottom in the MMT spectrum. We elected to not correct manually for this effect, but we note that this will likely result in underestimating the column density of the very strong lines in the MMT spectrum.

After extracting the spectra from individual orders, we performed the continuum fits to the spectra with the use of the FINDSL programme (Aldcroft et al. 1994). The spectra were then normalized, rebinned to common wavelength pixels and coadded, weighed with the variance.

Not surprisingly, the trickiest part of this part of the analysis was the continuum fits. The spectra are heavily absorbed bluewards of the Ly $\alpha$ emission line. To fit continua in the uncertain areas, we applied a technique similar to the one described in M03, i.e., utilizing the spectrum shapes in the unabsorbed orders. We note that this effect is less pronounced in our case, since a single echelle order in our spectra - which have lower resolution than the Subaru spectra - covers a larger wavelength range, providing for more possibilities to "anchor" the continuum.

However, the continuum fits remain uncertain in two particularly heavily blended areas. Comparison between continuum location in the normalized spectra from Keck and MMT shows that there remains a 15-20\% uncertainty in those regions. In one case, it is partly caused by the fact that one of the ESI echelle orders ends inside the blended region, forcing manual anchoring of the continuum fit. In the other case, it is the fact that the blended region is on the wing of the emission line, which coincides with the peak sensitivity of the echelle order; this results in a very uncertain shape of the continuum exactly where the technique of using shapes from unabsorbed regions cannot be applied. We will return to this issue in Sect. 3.1.1.

We note that our continuum fits generally appear to agree well with Misawa et al.'s analysis of their data. One notable exception will be discussed in more detail in Sect. 3.1.1.

The next step in the analysis - resolving and identifying absorption lines - was a very laborious process, since our spectra were heavily blended. In order to avoid bias, we performed the fits blindly, i.e., we decided not to use the information from Misawa et al. As a consequence, our results do differ quite significantly in some cases, especially in the number of individual components, into which some of the systems were resolved. However, since the primary goal of our analysis is to obtain information on the the column densities of as many ions as possible in order to use them as constraints for the modeling of the quasar intrinsic emission, the important information is the total column density in the system, and not the number of the individual components. In this regard, in the systems we have in common - primarily the vicinity of the C IV emission line - our results do qualitatively agree with those of M03 and M05.

The line fits were done using VPGUESS/VPFIT tools ${ }^{3}$. This programme fits multiple Voigt profiles, with absorption redshift $z_{\text {abs }}$, column density $N$ and Doppler parameter $b$ as the fitted parameters, and it is very well suited for analysis of blended spectra. However, our spectra are exceptionally crowded and in some

\footnotetext{
${ }^{3}$ http://www. eso.org/ jliske/vpguess/,
} http://www.ast.cam.ac.uk/ rfc/vpfit.html cases we had to use simplifying assumptions in order to prevent the programme from performing fits that were obviously nonphysical. We also had to apply some judgement calls. The cases where it was necessary are mentioned in the following subsections. We note that the absorption spectrum of HS1603+3820 is so complex and so severely blended that M03 and M05 had to resort to a similar approach, even in the Subaru spectrum, which had markedly higher resolution.

A large part of the work presented in M05 was devoted to the analysis of partial coverage of the QSO by the absorbers. It is clear that at least some of the absorbers in the vicinity of the quasar do not entirely cover the continuum emitting region. In our analysis, we accounted for this effect only in the one case where it was clearly necessary to obtain physically sound parameter fits - the thick C IV lines in System A (see Sect. 3.1.1), which were clearly saturated but did not reach zero intensity in the line bottoms. We note that M05 presented arguments indicating that other systems - including those with $z_{\mathrm{abs}} \ll z_{\mathrm{em}}-$ may also show partial coverage. We will discuss this during the description of the relevant systems.

The resolution of our spectra was slightly higher than the expected values of the Doppler parameter for heavy element lines. High resolution data from Subaru indicated that in many cases the metal lines had $b$ as low as $\sim 5 \mathrm{~km} \mathrm{~s}^{-1}$, while FWHM of 40-50 $\mathrm{km} \mathrm{s}^{-1}$ translates (for Gaussian lines) to $b \approx$ $25-30 \mathrm{~km} \mathrm{~s}^{-1}$. On the other hand, many lines have $b$ comparable to the spectrum resolution or higher. In our analysis of the spectra we thus used the iterative approach. We first freed all parameters in all fitted lines. Then for all lines where the resulting value of the Doppler parameter was significantly below the resolution, we fixed $b$ at the value that corresponded to the value of the parameter for other lines for which it was well established (using values for other metal lines wherever it was possible and hydrogen lines in other cases), scaled with the square root of the element's atomic mass, and re-ran the fit. In the situation where one or more lines had inconsistent values of the Doppler parameter, we investigated the possibility of having the line as a blend with other features.

It is relatively easy to fall into the trap of adding narrow lines in order to improve the fits of large features. We avoided this issue by adding lines only if it resulted in significant improvement of the fit, and all cases when this was done were individually examined.

In total, we found 49 individual absorption clouds, which can be grouped into eleven distinct "systems". We stress that the systems are defined based on their proximity in the spectrum, and the fact that two absorbers are considered members of the same system does not in principle mean that they are physically related.

In Tables 1-4 we show line lists from all systems detected in the spectra from Keck/ESI. In the tables, the first column shows the line designation; the first component refers to the separate cloud within the considered system, and the second part, after the dot, is the ordinal number of individual lines seen in this cloud. The second column gives the ion identification (ID). The following columns give the absorption redshift, the Doppler parameter, column density and (in Table 1) the covering factor, $C_{\mathrm{f}}$, as defined in Hamann et al. (1997). The last column gives (where available) the line identification in M05. We mark lines for which the derivation of parameters is made uncertain by blending and/or by the fact that the line is saturated. The line lists from the MMT/Blue Channel spectrum, as well as the entire Keck and MMT spectra, are available on the World Wide Web at http://alpha.uwb.edu.pl/mrk/hs1603/. In addition, in 
Table 1. Lines from System A $\left(z_{\mathrm{abs}}=2.44\right)$ detected in the Keck spectrum.

\begin{tabular}{|c|c|c|c|c|c|}
\hline No.Line ID & $z_{\text {abs }}$ & $\begin{array}{c}b \\
\left(\mathrm{~km} \mathrm{~s}^{-1}\right)\end{array}$ & $\begin{array}{l}\log N \\
\left(\mathrm{~cm}^{-2}\right)\end{array}$ & $\overline{\overline{C_{\mathrm{f}}}}$ & M05 ID \\
\hline $1 . \mathrm{HI}$ & $2.41941 \pm 0.00002$ & $62.58 \pm 6.51$ & $13.656 \pm 0.033$ & 0.61 & \\
\hline 1.C IV था & $2.41941 \pm 0.00002$ & $72.74 \pm 3.42$ & $14.965 \pm 0.044$ & 0.36 & $1 ; \mathrm{CIV}$ \\
\hline $1 . \mathrm{NV} \sharp$ & $2.41941 \pm 0.00002$ & $26.74 \pm 17.05$ & $13.235 \pm 0.964$ & & \\
\hline 1.Si IV & $2.41941 \pm 0.00002$ & 6.50 (fixed) & $12.065 \pm 0.182$ & & \\
\hline $2 . \mathrm{HI} \|$ & $2.42001 \pm 0.00002$ & 25.00 (fixed) & $14.496 \pm 0.087$ & 0.61 & \\
\hline 2.C IV II & $2.42001 \pm 0.00002$ & $198.64 \pm 21.32$ & $14.225 \pm 0.054$ & 0.36 & \\
\hline $2 . \mathrm{NV} \sharp$ & $2.42001 \pm 0.00002$ & 9.30 (fixed) & $13.008 \pm 0.257$ & & \\
\hline 2.SiIV & $2.42001 \pm 0.00002$ & 6.50 (fixed) & $11.789 \pm 0.325$ & & \\
\hline $3 . \mathrm{HI}$ & $2.42344 \pm 0.00025$ & 25.00 (fixed) & $13.132 \pm 0.037$ & 0.61 & \\
\hline 3.C IV II & $2.42344 \pm 0.00025$ & $61.09 \pm 18.07$ & $13.440 \pm 0.068$ & 0.36 & \\
\hline $3 . \mathrm{NV} \sharp$ & $2.42344 \pm 0.00025$ & 9.30 (fixed) & $12.683 \pm 0.319$ & & \\
\hline $4 . \mathrm{HI}$ & $2.42447 \pm 0.00019$ & 25.00 (fixed) & $13.131 \pm 0.037$ & 0.61 & \\
\hline 4.C IV & $2.42447 \pm 0.00019$ & $34.72 \pm 17.17$ & $13.090 \pm 0.333$ & 0.36 & $2 ; \mathrm{CIV}$ \\
\hline 4.N V \# & $2.42447 \pm 0.00019$ & 9.30 (fixed) & $13.296 \pm 0.349$ & & \\
\hline $5 . \mathrm{HI}$ & $2.42554 \pm 0.00002$ & 25.00 (fixed) & 12.420 (fixed) & 0.61 & \\
\hline 5.C IV & $2.42554 \pm 0.00002$ & 10.00 (fixed) & $14.313 \pm 0.870$ & 0.36 & $3 ; \mathrm{CIV}$ \\
\hline $5 . \mathrm{NV} \#$ & $2.42554 \pm 0.00002$ & 9.30 (fixed) & $12.885 \pm 0.106$ & & \\
\hline 5.Si III \# & $2.42554 \pm 0.00002$ & 6.50 (fixed) & $12.667 \pm 0.041$ & & \\
\hline $6 . \mathrm{HI} I$ & $2.42696 \pm 0.00003$ & 25.00 (fixed) & $14.700 \pm 0.050$ & 0.61 & \\
\hline 6.C IV & $2.42696 \pm 0.00003$ & $44.13 \pm 10.52$ & $13.322 \pm 0.086$ & 0.36 & \\
\hline $6 . \mathrm{NV} \sharp$ & (a) & & & & \\
\hline $7 . \mathrm{HI}$ & $2.42833 \pm 0.00001$ & 25.00 (fixed) & $13.941 \pm 0.016$ & & \\
\hline 7.C IV & $2.42833 \pm 0.00001$ & 10.00 (fixed) & $13.526 \pm 0.055$ & & \\
\hline 7.N V \# & $2.42833 \pm 0.00001$ & 9.30 (fixed) & $12.650 \pm 0.200$ & & \\
\hline $8 . \mathrm{HI}$ & $2.43509 \pm 0.00006$ & $49.08 \pm 30.79$ & $12.361 \pm 0.704$ & & \\
\hline 8.C IV & $2.43509 \pm 0.00006$ & $38.64 \pm 8.37$ & $13.440 \pm 0.058$ & & $4 ; \mathrm{CIV}$ \\
\hline $8 . \mathrm{NV} \sharp$ & $2.43509 \pm 0.00006$ & 9.30 (fixed) & $12.977 \pm 0.477$ & & \\
\hline 8.Si III $\sharp$ & $2.43509 \pm 0.00006$ & 6.50 (fixed) & $\leq 16.000$ & & \\
\hline 9.H I & $2.43710 \pm 0.00005$ & $100.56 \pm 33.64$ & $13.091 \pm 0.110$ & & \\
\hline 9.C IV & $2.43710 \pm 0.00005$ & $81.40 \pm 8.82$ & $13.970 \pm 0.024$ & & 5 ; C IV \\
\hline $9 . \mathrm{NV} \#$ & $2.43710 \pm 0.00005$ & 9.30 (fixed) & $13.094 \pm 1.220$ & & \\
\hline 9.Si III \# & $2.43710 \pm 0.00005$ & 6.50 (fixed) & $\leq 16.000$ & & \\
\hline 9.Si IV & $2.43710 \pm 0.00005$ & 6.50 (fixed) & $12.050 \pm 0.190$ & & \\
\hline $10 . \mathrm{H} \mathrm{I}$ & $2.43753 \pm 0.00002$ & 25.00 (fixed) & $13.262 \pm 0.061$ & & \\
\hline 10.C IV & $2.43753 \pm 0.00002$ & 10.00 (fixed) & $13.596 \pm 0.121$ & & 6; CIV \\
\hline $10 . \mathrm{N} \mathrm{V} \sharp$ & $2.43753 \pm 0.00002$ & 9.30 (fixed) & $13.717 \pm 0.327$ & & \\
\hline 10.Si III $\sharp$ & $2.43753 \pm 0.00002$ & 6.50 (fixed) & $\leq 16.000$ & & \\
\hline 10.Si IV & $2.43753 \pm 0.00002$ & 6.50 (fixed) & $11.839 \pm 0.292$ & & \\
\hline $11 . \mathrm{HI}$ & $2.43911 \pm 0.00030$ & 138.45 (fixed) & $13.244 \pm 0.087$ & & \\
\hline 11.C IV & $2.43911 \pm 0.00030$ & $50.59 \pm 40.10$ & $13.273 \pm 0.481$ & & 7; C IV \\
\hline $11 . \mathrm{N} \mathrm{V} \sharp$ & (a) & & & & \\
\hline 11.Si III $\sharp$ & $2.43911 \pm 0.00030$ & 6.50 (fixed) & $\leq 15.376$ & & \\
\hline $12 . \mathrm{H} \mathrm{I}$ & $2.43989 \pm 0.00019$ & 25.00 (fixed) & $12.464 \pm 0.344$ & & \\
\hline 12.C IV & $2.43989 \pm 0.00019$ & 10.00 (fixed) & $12.825 \pm 0.534$ & & CIV \\
\hline $12 . \mathrm{NV} \sharp$ & $2.43989 \pm 0$ & 9.30 (fixed) & $12.485 \pm 0.880$ & & \\
\hline 12.Si III $\sharp$ & $2.43989 \pm 0.00019$ & 6.50 (fixed) & $\leq 16.000$ & & \\
\hline $13 . \mathrm{HI}$ & $2.44079 \pm 0.00042$ & 72.42 (fixed) & $13.057 \pm 0.103$ & & \\
\hline 13.C IV & $2.44079 \pm 0.00042$ & $77.05 \pm 50.41$ & $13.518 \pm 1.886$ & & 9; C IV \\
\hline $13 . \mathrm{N} \mathrm{V} \sharp$ & (a) & & & & \\
\hline 13.Si III $\sharp$ & $2.44079 \pm 0.00042$ & 6.50 (fixed) & $\leq 12.734$ & & \\
\hline $14 . \mathrm{HI}$ & $2.44215 \pm 0.00030$ & 25.00 (fixed) & $13.004 \pm 0.131$ & & \\
\hline 14.C IV & $2.44215 \pm 0.00030$ & 10.00 (fixed) & $13.787 \pm 0.100$ & & 10 ; C IV \\
\hline $14 . \mathrm{N} \mathrm{V} \sharp$ & $2.44215 \pm 0.00030$ & 9.30 (fixed) & $14.054 \pm 0.101$ & & \\
\hline 14.Si III $\sharp$ & $2.44215 \pm 0.00030$ & 6.50 (fixed) & $\leq 11.587$ & & \\
\hline $15 . \mathrm{H} \mathrm{I}$ & $2.44228 \pm 0.00021$ & $81.41 \pm 43.00$ & $13.106 \pm 0.221$ & & \\
\hline 15.C IV & $2.44228 \pm 0.00021$ & $123.22 \pm 87.76$ & $13.240 \pm 0.305$ & & $11 ;$ C IV \\
\hline $15 . \mathrm{N} \mathrm{V} \sharp$ & $2.44228 \pm 0.00021$ & 9.30 (fixed) & $13.808 \pm 0.171$ & & \\
\hline 15.Si III $\sharp$ & $2.44228 \pm 0.00021$ & 6.50 (fixed) & $\leq 11.520$ & & \\
\hline
\end{tabular}

Table 5 we list the total column densities of all ions detected in all systems and compare them to the values found in the Subaru data. The following section contains more detailed discussion of the individual systems.

\subsection{Systems at $Z_{\mathrm{abs}} \approx Z_{\mathrm{em}}$}

Of the eleven distinct systems, four (A-D) are close to the emission redshift of the quasar. The ejection velocities range 
Table 1. continued.

\begin{tabular}{|c|c|c|c|c|c|}
\hline No.Line ID & $\overline{z_{\mathrm{abs}}}$ & $\begin{array}{c}b \\
\left(\mathrm{~km} \mathrm{~s}^{-1}\right)\end{array}$ & $\begin{array}{l}\log N \\
\left(\mathrm{~cm}^{-2}\right)\end{array}$ & $C_{\mathrm{f}}$ & M05 ID \\
\hline $16 . \mathrm{HI}$ & $2.44325 \pm 0.00148$ & 114.39 (fixed) & $13.086 \pm 0.203$ & & \\
\hline 16.C IV & $2.44325 \pm 0.00148$ & 100.74 (fixed) & $13.709 \pm 0.055$ & & 12; C IV \\
\hline $16 . \mathrm{N} \mathrm{V} \sharp$ & (a) & & & & \\
\hline 16.Si III $\sharp$ & $2.44325 \pm 0.00148$ & 6.50 (fixed) & $\leq 12.394$ & & \\
\hline 17.H I & $2.44480 \pm 0.00018$ & $91.30 \pm 64.73$ & $13.093 \pm 0.104$ & & \\
\hline 17.C IV & $2.44480 \pm 0.00018$ & $30.00 \pm 20.54$ & $13.070 \pm 0.176$ & & 14; C IV \\
\hline $17 . \mathrm{N} \mathrm{V} \#$ & $2.44480 \pm 0.00018$ & 9.30 (fixed) & $13.094 \pm 0.433$ & & \\
\hline 17.Si III $\sharp$ & $2.44480 \pm 0.00018$ & 6.50 (fixed) & $\leq 12.679$ & & \\
\hline $18 . \mathrm{HI}$ & $2.44641 \pm 0.00021$ & $75.27 \pm 25.93$ & $13.154 \pm 0.216$ & & \\
\hline 18.C IV & $2.44641 \pm 0.00021$ & $109.27 \pm 58.99$ & $13.807 \pm 0.202$ & & 15; C IV \\
\hline $18 . \mathrm{N} \mathrm{V} \sharp$ & (a) & & & & \\
\hline 18.Si III $\sharp$ & $2.44641 \pm 0.00021$ & 6.50 (fixed) & $\leq 13.483$ & & \\
\hline 19.H I & $2.44818 \pm 0.00002$ & 25.00 (fixed) & $13.594 \pm 0.020$ & & \\
\hline 19.C IV & $2.44818 \pm 0.00002$ & $41.51 \pm 49.68$ & $12.920 \pm 0.366$ & & \\
\hline $19 . \mathrm{N} \mathrm{V} \sharp$ & (a) & & & & \\
\hline 19.Si III $\sharp$ & $2.44818 \pm 0.00002$ & 6.50 (fixed) & $\leq 12.467$ & & \\
\hline $20 . \mathrm{HI}$ & $2.44954 \pm 0.00035$ & 41.27 (fixed) & 12.608 (fixed) & & \\
\hline 20.C IV & $2.44954 \pm 0.00035$ & $62.77 \pm 55.72$ & $13.133 \pm 0.388$ & & \\
\hline $20 . \mathrm{N} \mathrm{V} \sharp$ & (a) & & & & \\
\hline 20.Si III \# & $2.44954 \pm 0.00035$ & 6.50 (fixed) & $\leq 12.644$ & & \\
\hline 21.H I & $2.45069 \pm 0.00082$ & 25.00 (fixed) & 12.874 (fixed) & & \\
\hline 21.C IV & $2.45069 \pm 0.00082$ & $65.13 \pm 108.68$ & $13.117 \pm 0.681$ & & \\
\hline 21.N V \# & $2.45069 \pm 0.00082$ & 9.30 (fixed) & $11.928 \pm 1.265$ & & \\
\hline 21.Si III $\sharp$ & $2.45069 \pm 0.00082$ & 6.50 (fixed) & $\leq 12.511$ & & \\
\hline 22.H I II & $2.45174 \pm 0.00041$ & 51.00 (fixed) & 14.245 (fixed) & & \\
\hline 22.C IV & $2.45174 \pm 0.00041$ & $53.88 \pm 44.25$ & $13.059 \pm 0.340$ & & \\
\hline $22 . \mathrm{N} \mathrm{V} \sharp$ & $2.45174 \pm 0.00041$ & 9.30 (fixed) & $12.686 \pm 0.923$ & & \\
\hline 22.Si III $\sharp$ & $2.45174 \pm 0.00041$ & 6.50 (fixed) & $\leq 14.411$ & & \\
\hline
\end{tabular}

$\sharp$ - Heavy-element line in the Ly $\alpha$ forest region of the spectrum; II - saturated line; (a) - detected in the MMT spectrum, but not seen in the Keck spectrum.

from $\sim 10000 \mathrm{~km} \mathrm{~s}^{-1}$ for System A to negative (i.e. $z_{\mathrm{abs}}>z_{\mathrm{em}}$ ) $\sim 1200 \mathrm{~km} \mathrm{~s}^{-1}$ for System D. Figure 2 shows the Keck spectrum of HS1603+3820 in the vicinity of the emission redshift of the quasar for several absorbers. One can clearly see that some of the systems contain lines from several species.

\subsubsection{System $A, z_{a b s}=2.44$}

Five heavy-element absorbers were recognized in this system in the medium-resolution data of D99. At least one of the components, at $z_{\mathrm{abs}}=2.4189$, was identified as associated, since the $\mathrm{C} \operatorname{IV}(\lambda 1548) / \mathrm{C} \operatorname{IV}(\lambda 1550)$ ratio was 1 , indicating that the cloud was optically thick, yet the lines did not reach zero intensity in their bottoms, suggesting that the cloud in which the lines originated did not obscure the entire continuum emitting source.

M03 decomposed this system into seven individual components, while M05 increased this number to fifteen systems. Their analysis also confirmed that the absorbers do not appear to entirely cover the quasar, and they found indication that this system is variable. These findings were confirmed and expanded in the recent paper (M07).

System A is unambiguously associated with HS1603+3820, and at the same time it has a large relative velocity with respect to the quasar (ranging from $\sim 7500$ to $\sim 10000 \mathrm{~km} \mathrm{~s}^{-1}$ ), which means that it is almost certainly located very close to the quasar central engine and its properties are very likely determined by the radiation from the quasar. This system is thus the ideal candidate for the modeling of the quasar continuum.
In our analysis, we found twenty two components belonging to System A. The results are listed in Table 1; we show the Ly $\alpha$ and C IV lines in Fig. 4. For the thick absorbers in the blue end of the system we applied the correction for partial covering using empirically determined covering factors (listed in Table 1). We estimate the uncertainty of the covering factor to be of the order of $\sim 10 \%$; the values agree qualitatively with the values found in the Subaru data.

Our results for System A cannot be directly compared with the results from Subaru, since at least four of the components seen in our spectra (Nos. 19-22 in Table 1) result from the difference between the continuum fits in both sets of data.

In their analysis, Misawa et al. (also in their very recent paper) treated the wavelength region between $\sim 5345$ and $\sim 5360 \AA$ as unabsorbed (see Fig. 4 in M05 or Fig. 2 in M07). However, this region clearly contains absorption in both the Keck and MMT data. In Fig. 3 we show the raw spectra of this region from both the Keck and MMT observations. In both cases one can clearly see that the continuum does drop bluewards of $\sim 5360 \AA$, and we had to fit lines in this region in order to account for this drop. This was actually a difficult task, since it was hard to resolve the dip into individual systems. We primarily relied on the information from the Ly $\alpha$ region, where strong, but heavily blended absorption was present. We found that at least four systems (i.e. Nos. 19-22) with reasonable properties are necessary to satisfactorily fit this region. However, while we are confident that the absorbers are present in this region, we have to treat their derived physical parameters as somewhat uncertain. We note that if the derived densities are taken at face value, then 
Table 2. Lines from System B $\left(z_{\text {abs }}=2.48\right)$ detected in the Keck spectrum.

\begin{tabular}{|c|c|c|c|c|}
\hline No.Line ID & $z_{\text {abs }}$ & $\begin{array}{c}b \\
\left(\mathrm{~km} \mathrm{~s}^{-1}\right)\end{array}$ & $\begin{array}{l}\log N \\
\left(\mathrm{~cm}^{-2}\right)\end{array}$ & M05 ID \\
\hline 1.HI I & $2.47723 \pm 0.00002$ & 25.00 (fixed) & $14.583 \pm 0.228$ & \\
\hline 1.C IV & $2.47723 \pm 0.00002$ & 10.00 (fixed) & $14.223 \pm 0.141$ & 1, 2; C IV \\
\hline 1.C II & $2.47723 \pm 0.00002$ & 10.00 (fixed) & $13.320 \pm 0.093$ & $1, \mathrm{C}$ II \\
\hline $1 . \mathrm{NV} \sharp$ & $2.47723 \pm 0.00002$ & 9.30 (fixed) & $13.617 \pm 0.426$ & \\
\hline 1.Mg II & $2.47723 \pm 0.00002$ & 7.00 (fixed) & $12.123 \pm 0.300$ & \\
\hline 1.Al III & $2.47723 \pm 0.00002$ & 6.70 (fixed) & $11.923 \pm 0.469$ & \\
\hline 1.Al II & $2.47723 \pm 0.00002$ & 6.70 (fixed) & $11.020 \pm 1.288$ & \\
\hline 1.Si IV & $2.47723 \pm 0.00002$ & 6.50 (fixed) & $13.258 \pm 0.082$ & 1, 2; Si IV \\
\hline 1.Si III $\sharp$ & $2.47723 \pm 0.00002$ & 6.50 (fixed) & $13.068 \pm 1.434$ & \\
\hline 1.Si II & $2.47723 \pm 0.00002$ & 6.50 (fixed) & $12.554 \pm 0.103$ & 1, 2; Si II \\
\hline 2.H I & $2.47762 \pm 0.00004$ & 25.00 (fixed) & $14.000 \pm 0.154$ & \\
\hline 2.C IV & $2.47762 \pm 0.00004$ & 10.00 (fixed) & $13.643 \pm 0.110$ & 3-5; C IV \\
\hline $2 . \mathrm{NV} \#$ & $2.47762 \pm 0.00004$ & 9.30 (fixed) & $12.626 \pm 0.645$ & \\
\hline 2.Mg II & $2.47762 \pm 0.00004$ & 7.00 (fixed) & $11.915 \pm 0.416$ & \\
\hline 2.Al III & (a) & & & \\
\hline 2.Al II & (a) & & & \\
\hline 2.Si III $\sharp$ & $2.47762 \pm 0.00004$ & 6.50 (fixed) & $16.616 \pm 0.333$ & 1; Si III \\
\hline 2.Si II & (a) & & & \\
\hline 3.H I II & $2.47847 \pm 0.00001$ & 25.00 (fixed) & $14.625 \pm 0.100$ & \\
\hline 3.C IV & $2.47847 \pm 0.00001$ & 10.00 (fixed) & $15.265 \pm 0.135$ & 6-10; C IV \\
\hline 3.C II & (a) & & & $2 ; \mathrm{C}$ II \\
\hline $3 . \mathrm{NV} \#$ & $2.47847 \pm 0.00001$ & 9.30 (fixed) & $13.493 \pm 0.072$ & 1,$2 ; \mathrm{N} \mathrm{V}$ \\
\hline 3.Al III & (a) & & & \\
\hline 3.Al II & (a) & & & \\
\hline 3.Si IV & $2.47847 \pm 0.00001$ & $26.40 \pm 3.23$ & $12.827 \pm 0.077$ & 3, 4; Si IV \\
\hline 3.Si III $\sharp$ & $2.47847 \pm 0.00001$ & $26.40 \pm 3.23$ & $14.203 \pm 0.206$ & 2; Si III \\
\hline 3.Si II & (a) & & & 3, 4; Si II \\
\hline 4.HIII & $2.47938 \pm 0.00001$ & 25.00 (fixed) & $15.810 \pm 1.099$ & \\
\hline 4.C IV & $2.47938 \pm 0.00001$ & $29.97 \pm 1.36$ & $14.515 \pm 0.026$ & 11-13; C IV \\
\hline 4.C II & $2.47938 \pm 0.00001$ & $29.97 \pm 1.36$ & $14.671 \pm 0.029$ & 3,$4 ;$ C II \\
\hline $4 . \mathrm{N} \mathrm{V} \sharp, \dagger$ & $2.47938 \pm 0.00001$ & $38.37 \pm 30.95$ & $13.512 \pm 0.087$ & $3,4,5 ; \mathrm{N} \mathrm{V}$ \\
\hline $4 . \mathrm{OI} \ddagger$ & $2.47938 \pm 0.00001$ & 8.70 (fixed) & $13.754 \pm 0.184$ & $1 ;$ O I \\
\hline 4.Mg II † & $2.47938 \pm 0.00001$ & 7.00 (fixed) & $15.096 \pm 0.159$ & \\
\hline $4 . \mathrm{Mg} \mathrm{I}$ & $2.47938 \pm 0.00001$ & 7.00 (fixed) & $11.766 \pm 0.209$ & \\
\hline 4.Al III & $2.47938 \pm 0.00001$ & 6.70 (fixed) & $13.054 \pm 0.107$ & \\
\hline 4.Al II & $2.47938 \pm 0.00001$ & 6.70 (fixed) & $14.518 \pm 0.239$ & 1, 2; Al II \\
\hline 4.SiIV & $2.47938 \pm 0.00001$ & $21.87 \pm 5.96$ & $13.398 \pm 0.035$ & 5, 6; Si IV \\
\hline 4.Si III $\sharp$ & $2.47938 \pm 0.00001$ & $21.87 \pm 5.96$ & $13.810 \pm 0.120$ & 3; Si III \\
\hline 4.Si II & $2.47938 \pm 0.00001$ & $21.87 \pm 5.96$ & $13.938 \pm 0.151$ & 5, 6; Si II \\
\hline 4.Fe III $\sharp \dagger$ & (b) & & & \\
\hline 4.Fe II $\sharp$ & $2.47938 \pm 0.00001$ & 4.65 (fixed) & 13.250 (fixed) & $1 ; \mathrm{Fe}$ II \\
\hline $5 . \mathrm{HI} \mathbb{I}$ & $2.47998 \pm 0.00002$ & 25.00 (fixed) & $14.110 \pm 0.503$ & \\
\hline 5.C IV & $2.47998 \pm 0.00002$ & 10.00 (fixed) & $14.550 \pm 0.080$ & 14, 15; C IV \\
\hline $5 . \mathrm{NV} \sharp$ & $2.47998 \pm 0.00002$ & 9.30 (fixed) & $12.843 \pm 0.296$ & $6 ; \mathrm{N} \mathrm{V}$ \\
\hline 5.Al III & $2.47998 \pm 0.00002$ & 6.70 (fixed) & $11.836 \pm 0.573$ & \\
\hline 5.Al II & $2.47998 \pm 0.00002$ & 6.70 (fixed) & $11.517 \pm 0.484$ & 3; Al II \\
\hline 5.Si IV & $2.47998 \pm 0.00002$ & $34.32 \pm 5.42$ & $13.075 \pm 0.067$ & 7; Si IV \\
\hline 5.Si III $\sharp$ & $2.47998 \pm 0.00002$ & $34.32 \pm 5.42$ & $12.984 \pm 0.065$ & 4; Si III \\
\hline 5.Si II & $2.47998 \pm 0.00002$ & $34.32 \pm 5.42$ & $11.355 \pm 1.345$ & 7; Si II \\
\hline 5.Fe III $\sharp$ & (b) & & & \\
\hline 5.Fe II $\sharp$ & $2.47998 \pm 0.00002$ & 4.65 (fixed) & $12.843 \pm 0.214$ & \\
\hline $6 . \mathrm{HI} I$ & $2.48064 \pm 0.00002$ & 25.00 (fixed) & $14.969 \pm 0.590$ & \\
\hline 6.C IV & $2.48064 \pm 0.00002$ & 10.00 (fixed) & $14.110 \pm 0.076$ & 16, 17; C IV \\
\hline 6.C II & $2.48064 \pm 0.00002$ & 10.00 (fixed) & $13.983 \pm 0.053$ & 6, 7; C II \\
\hline $6 . \mathrm{NV} \#$ & $2.48064 \pm 0.00002$ & 9.30 (fixed) & $12.423 \pm 0.371$ & \\
\hline 6.Mg II & $2.48064 \pm 0.00002$ & 7.00 (fixed) & $12.903 \pm 0.115$ & \\
\hline 6.Al III & $2.48064 \pm 0.00002$ & 6.70 (fixed) & $12.061 \pm 0.362$ & \\
\hline 6.Al II & $2.48064 \pm 0.00002$ & 6.70 (fixed) & $11.968 \pm 0.199$ & 4 ; Al II \\
\hline 6.Si IV & $2.48064 \pm 0.00002$ & 6.50 (fixed) & $13.334 \pm 0.094$ & 8, 9; Si IV \\
\hline
\end{tabular}


Table 2. continued.

\begin{tabular}{|c|c|c|c|c|}
\hline No.Line ID & $z_{\mathrm{abs}}$ & $\begin{array}{c}b \\
\left(\mathrm{~km} \mathrm{~s}^{-1}\right)\end{array}$ & $\begin{array}{l}\log N \\
\left(\mathrm{~cm}^{-2}\right)\end{array}$ & $\overline{\mathrm{M} 05 \mathrm{ID}}$ \\
\hline $6 . S i$ III $\sharp, \dagger$ & $2.48064 \pm 0.00002$ & 6.50 (fixed) & $15.883 \pm 0.058$ & 5-7; Si III \\
\hline 6.Si II & $2.48064 \pm 0.00002$ & 6.50 (fixed) & $13.136 \pm 0.122$ & 8; Si II \\
\hline 6.Fe III \# & (b) & & & \\
\hline $7 . \mathrm{HI} I$ & $2.48145 \pm 0.00002$ & 25.00 (fixed) & $14.431 \pm 0.338$ & \\
\hline 7.C IV & $2.48145 \pm 0.00002$ & 10.00 (fixed) & $13.979 \pm 0.072$ & 18,$19 ;$ C IV \\
\hline 7.C II & $2.48145 \pm 0.00002$ & 10.00 (fixed) & $13.914 \pm 0.052$ & 8; C II \\
\hline 7.Nv $\sharp$ & $2.48145 \pm 0.00002$ & 9.30 (fixed) & $12.423 \pm 0.371$ & \\
\hline 7.Mg II & $2.48145 \pm 0.00002$ & 7.00 (fixed) & $13.027 \pm 0.112$ & \\
\hline 7.Al III & $2.48145 \pm 0.00002$ & 6.70 (fixed) & $12.562 \pm 0.150$ & \\
\hline 7. $\mathrm{Al} \mathrm{II}$ & $2.48145 \pm 0.00002$ & 6.70 (fixed) & $12.068 \pm 0.174$ & 5; $\mathrm{Al} \mathrm{II}$ \\
\hline 7.Si IV & $2.48145 \pm 0.00002$ & 6.50 (fixed) & $13.407 \pm 0.082$ & 10; Si IV \\
\hline 7.Si III $\sharp$ & $2.48145 \pm 0.00002$ & 6.50 (fixed) & $13.936 \pm 0.171$ & 8; Si III \\
\hline 7.Si II & $2.48145 \pm 0.00002$ & 6.50 (fixed) & 13.449 (fixed) & 9; Si II \\
\hline 7.Fe III $\sharp$ & (b) & & & \\
\hline 7.Fe II $\sharp$ & $2.48145 \pm 0.00002$ & 4.65 (fixed) & $12.526 \pm 0.293$ & \\
\hline $8 . \mathrm{HI} I$ & $2.48226 \pm 0.00002$ & 25.00 (fixed) & $15.111 \pm 0.100$ & \\
\hline 8.C IV & $2.48226 \pm 0.00002$ & 10.00 (fixed) & $12.537 \pm 0.407$ & \\
\hline $8 . \mathrm{C} \mathrm{II} \dagger$ & $2.48226 \pm 0.00002$ & 10.00 (fixed) & $14.374 \pm 0.072$ & 9-11; C II \\
\hline $8 . \mathrm{NV} \sharp$ & (a) & & & \\
\hline $8.0 \mathrm{I}$ & $2.48226 \pm 0.00001$ & 8.70 (fixed) & $13.549 \pm 0.066$ & \\
\hline 8.Mg II & $2.48226 \pm 0.00002$ & 7.00 (fixed) & $13.014 \pm 0.106$ & \\
\hline 8.Al III & $2.48226 \pm 0.00002$ & 6.70 (fixed) & $11.751 \pm 0.670$ & \\
\hline 8.Al II & $2.48226 \pm 0.00002$ & 6.70 (fixed) & $11.977 \pm 0.197$ & 6, 7; Al II \\
\hline 8.Si IV & (a) & & & \\
\hline 8.Si III \# & $2.48226 \pm 0.00002$ & 6.50 (fixed) & $13.124 \pm 0.113$ & 9, 10; Si III \\
\hline 8.Si II & $2.48226 \pm 0.00002$ & 6.50 (fixed) & $13.822 \pm 0.155$ & 10, 11; Si II \\
\hline
\end{tabular}

$\sharp-$ Heavy-element line in the Ly $\alpha$ forest region of the spectrum; $\neq-$ line blended by identified line (O I line is blended by C IV from System J); $\dagger-$ line is probably blended with an unidentified line; $\mathbb{I}$ - saturated line; (a) - detected in the MMT spectrum, but not seen in the Keck spectrum;

(b) - detected in the MMT spectrum, but noise is too strong in order to fit this line in the Keck spectrum.

Table 3. Lines from Systems C and D detected in the Keck spectrum.

\begin{tabular}{|c|c|c|c|c|}
\hline No.Line ID & $z_{\mathrm{abs}}$ & $\begin{array}{c}b \\
\left(\mathrm{~km} \mathrm{~s}^{-1}\right)\end{array}$ & $\begin{array}{l}\log N \\
\left(\mathrm{~cm}^{-2}\right)\end{array}$ & $\overline{\mathrm{M} 05 \mathrm{ID}}$ \\
\hline \multicolumn{5}{|c|}{ System C $z_{\text {abs }}=2.54$} \\
\hline 1. H I II & $2.53787 \pm 0.00003$ & 25.00 (fixed) & $15.659 \pm 0.270$ & \\
\hline 1.C IV & $2.53787 \pm 0.00003$ & $8.32 \pm 4.25$ & $13.510 \pm 0.155$ & 1; C IV \\
\hline \multicolumn{5}{|c|}{ System D zabs $=2.55$} \\
\hline 1.H I & $2.55222 \pm 0.00001$ & 25.00 (fixed) & $13.569 \pm 0.697$ & \\
\hline 1.C IV & $2.55222 \pm 0.00001$ & 10.00 (fixed) & $12.585 \pm 0.410$ & $1 ;$ C IV \\
\hline 2.H I & $2.55383 \pm 0.00015$ & 25.00 (fixed) & $14.414 \pm 1.474$ & \\
\hline 2.C IV & $2.55383 \pm 0.00015$ & 10.00 (fixed) & $13.456 \pm 0.790$ & 2, C IV \\
\hline $3 . \mathrm{HI} I$ & $2.55409 \pm 0.00018$ & 25.00 (fixed) & $16.042 \pm 1.916$ & \\
\hline 3.C IVII & $2.55409 \pm 0.00018$ & $14.45 \pm 7.54$ & $14.407 \pm 0.863$ & 3 ; C IV \\
\hline 3.Si IV & $2.55409 \pm 0.00018$ & $11.81 \pm 8.00$ & $12.850 \pm 0.043$ & 1; Si IV \\
\hline $4 . \mathrm{HI} I$ & $2.55442 \pm 0.00005$ & 25.00 (fixed) & $14.865 \pm 1.732$ & \\
\hline 4.C IVII & $2.55442 \pm 0.00005$ & $11.40 \pm 3.96$ & $15.200 \pm 1.134$ & 4 ; C IV \\
\hline 4.Si IV & $2.55442 \pm 0.00005$ & $10.64 \pm 4.40$ & $13.593 \pm 0.085$ & 2; Si IV \\
\hline 4.N V \# & $2.55442 \pm 0.00005$ & $38.57 \pm 16.44$ & $13.191 \pm 0.105$ & \\
\hline
\end{tabular}

II - Saturated line; \# - likely blend of components 3 and 4 .

the four systems contain of the order $20 \%$ of all H I column density in System A, but only $2 \%$ of the C IV column density; this would suggest that the presence of those clouds in the vicinity of System A is coincidental and there is no physical relation between them and the system absorbers.

The other place in the System A area where there is a difference between our analysis and the results from M05 is the $\sim 5308-5317 \AA$ region. This area contains the $\operatorname{Si} \mathrm{II}(\lambda 1527) \mathrm{ab}-$ sorption from System B (it is the feature near $\lambda \approx 5312 \AA$ in Fig. 4). We obtained a very good fit using eight lines, compared with 11 lines used in M05. We note, however, that in the earlier analysis M03 also used 8 lines.

Even though it is a heavily blended region, our analysis was aided by the fact that our spectra cover large wavelength ranges and contain other Si II lines from System B, which we could use to constrain the properties of the $\lambda 1527$ transition. Those lines clearly indicated that this line is not as strong as described in M05. However, this also indicated that this region had to contain additional $\mathrm{C}$ IV lines. We added new C IV doublets, which are labeled with 5, 6 and 7 in Fig. 4 and Table 1, and we also 
Table 4. Lines from Systems E-K detected in the Keck spectrum.

\begin{tabular}{|c|c|c|c|c|}
\hline No.Line ID & $z_{\mathrm{abs}}$ & $\begin{array}{c}b \\
\left(\mathrm{~km} \mathrm{~s}^{-1}\right) \\
\end{array}$ & $\begin{array}{l}\log N \\
\left(\mathrm{~cm}^{-2}\right) \\
\end{array}$ & M05 ID \\
\hline \multicolumn{5}{|c|}{ System E $z_{\text {abs }}=1.89$} \\
\hline 1.H I & (a) & & & \multirow{3}{*}{$1 ;$ C IV } \\
\hline $1 . \mathrm{CIV}$ & $1.88805 \pm 0.00001$ & 10.00 (fixed) & $13.873 \pm 0.032$ & \\
\hline 1.Si IV $\sharp$ & $1.88805 \pm 0.00001$ & 6.50 (fixed) & $13.036 \pm 0.064$ & \\
\hline $2 . \mathrm{HI}$ & (a) & \multirow{3}{*}{10.00 (fixed) } & & \multirow{3}{*}{$2 ; \mathrm{CIV}$} \\
\hline 2.C IV & $1.88851 \pm 0.00007$ & & $13.576 \pm 0.034$ & \\
\hline 2.SiIV \# & 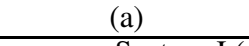 & & & \\
\hline \multicolumn{5}{|c|}{$\begin{array}{l}\text { System J (new system) } z_{\text {abs }}=1.93 \\
\text { (a) }\end{array}$} \\
\hline $2 . \mathrm{CIV} \ddagger$ & $1.92658 \pm 0.00001$ & 10.00 (fixed) & $13.727 \pm 0.081$ & \\
\hline \multicolumn{5}{|c|}{ System F $z_{\text {abs }}=1.97$} \\
\hline 1.H I & (a) & & & \\
\hline 1.C IV & $1.96399 \pm 0.00002$ & 10.00 (fixed) & $13.699 \pm 0.213$ & $1 ; \mathrm{C}$ IV \\
\hline 2.H I & (a) & & & \\
\hline 2.C IV & $1.96420 \pm 0.00002$ & 10.00 (fixed) & $14.185 \pm 0.179$ & 2, 3; C IV \\
\hline $3 . \mathrm{HI}$ & (a) & & & \\
\hline 3.C IV & $1.96519 \pm 0.00001$ & 10.00 (fixed) & $14.241 \pm 0.030$ & 4, 5; C IV \\
\hline 4.H I & (a) & & & \\
\hline 4.C IV & $1.96572 \pm 0.00001$ & 10.00 (fixed) & $13.574 \pm 0.026$ & 6; C IV \\
\hline \multicolumn{5}{|c|}{ System G $z_{\mathrm{abs}}=2.07$} \\
\hline 1.H I & (a) & & & \\
\hline 1.C IV & $2.07061 \pm 0.00005$ & 10.00 (fixed) & $14.385 \pm 0.576$ & $1 ;$ C IV \\
\hline 1.Si IV \# & $2.07061 \pm 0.00005$ & 6.50 (fixed) & $12.887 \pm 0.307$ & $1 ;$ Si IV \\
\hline 2.H I & (a) & & & \\
\hline 2.C IV & $07091 \pm 0.00005$ & 10.00 (fixed) & $14.487 \pm 0.575$ & 2; C IV \\
\hline 2.SiIV $\sharp$ & $2.07091 \pm 0.00005$ & 6.50 (fixed) & $13.491 \pm 0.188$ & 2; Si IV \\
\hline \multicolumn{5}{|c|}{ System I $z_{\text {abs }}=2.18$} \\
\hline $1 . \mathrm{H} \mathrm{I}$ & (a) & & & \\
\hline 1.C IV & $2.17560 \pm 0.00002$ & 10.00 (fixed) & $13.295 \pm 0.036$ & \\
\hline 1.Si IV & $2.17560 \pm 0.00002$ & 6.50 (fixed) & $12.359 \pm 0.069$ & \\
\hline 2.H I & (a) & & & \\
\hline 2.C IV & $2.17606 \pm 0.00005$ & 10.00 (fixed) & $13.066 \pm 0.056$ & \\
\hline 2.Si IV & $2.17606 \pm 0.00005$ & 6.50 (fixed) & $12.107 \pm 0.123$ & \\
\hline $3 . \mathrm{HI}$ & (a) & & & \\
\hline 3.C IV & $2.17645 \pm 0.00001$ & 10.00 (fixed) & $13.741 \pm 0.033$ & \\
\hline 3.Si IV & $2.17645 \pm 0.00001$ & 6.50 (fixed) & $12.750 \pm 0.040$ & \\
\hline \multicolumn{5}{|c|}{ System K (new system) $z_{\text {abs }}=2.26$} \\
\hline 1.H I II & $2.26214 \pm 0.00005$ & 25.00 (fixed) & $14.850 \pm 0.615$ & \\
\hline 1.C IV & $2.26214 \pm 0.00005$ & 10.00 (fixed) & $12.827 \pm 0.332$ & \\
\hline \multicolumn{5}{|c|}{ System $\mathrm{H} z_{\mathrm{abs}}=2.27$} \\
\hline $1 . \mathrm{HI} I$ & $2.26613 \pm 0.00002$ & 25.00 (fixed) & $14.075 \pm 0.570$ & \\
\hline 1.C IV & $2.26613 \pm 0.00002$ & 10.00 (fixed) & $13.200 \pm 0.035$ & $1 ; \mathrm{C}$ IV \\
\hline 1.Si IV & $2.26613 \pm 0.00002$ & 6.50 (fixed) & $12.527 \pm 0.053$ & \\
\hline 1.Si III $\sharp$ & $2.26613 \pm 0.00002$ & 6.50 (fixed) & $12.784 \pm 0.085$ & 1; Si III \\
\hline
\end{tabular}

$\sharp-$ Heavy-element line in Ly $\alpha$ forest region of the spectrum; $\$-$ line blended with identified line (C IV in System J is blended with O I from System B); II - saturated line; (a) - H I lines are not observable in Systems E, J, F, I and G, in either MMT or Keck spectra. H I lines in System I and 2.Si IV lines in System E are seen in the MMT spectrum.

added line No. 3. Both spectra, and especially the Keck spectrum, show that these doublets are required. Additionally, we split M05's line No. 1 into two lines - our doublets Nos. 1 and 2. This significantly improved our fit.

In System A, we also identified the Si IV, Si III, and N V lines. We show the Si IV lines in Fig. 5; the lines from the latter two ions are in the heavily crowded Ly $\alpha$ forest region. We note that the Si IV absorption was not seen in Misawa et al.'s data, even in the spectrum generated by coadding all the Subaru spectra (M07). This is clearly because of the high signal-to-noise ratio in our spectra.

We note that the components in System A have a rather large value of the $\mathrm{CIV} / \mathrm{H}$ I column density ratio, higher than 1 in virtually all cases and reaching $\sim 20$ in the extreme case. This is seen in both Keck (Table 1) and MMT spectra, and has strong implications for the modeling of the intrinsic spectrum of the quasar (Paper II). This effect is not seen in other systems, where practically all components have $N_{\mathrm{C} \text { IV }} / N_{\mathrm{HI}}<1$.

Our determination of the C IV column density agrees well with the value obtained in the Subaru data (Table 5). As mentioned earlier, none of the other ions seen in our spectra were seen in Misawa et al.'s data.

Finally, we would like to confirm the suspicion first expressed in M03 that the feature at $5527 \AA$ in the D99 spectrum is not a Fe II $(21608)$ line at $z_{\mathrm{abs}}=2.4367$. This feature is not present in our Keck and MMT data, and a close examination of the raw 1997 data revealed that it was in fact an artifact caused by a cosmic ray hit. 
Table 5. Total column densities of ions detected in the Keck spectrum.

\begin{tabular}{|c|c|c|c|}
\hline Ion & $\begin{array}{c}\log N_{\text {tot }}(\text { this paper }) \\
\left(\mathrm{cm}^{-2}\right)\end{array}$ & $\begin{array}{c}\log N_{\text {tot }}(\mathrm{M} 05) \\
\left(\mathrm{cm}^{-2}\right)\end{array}$ & $\begin{array}{c}\log N_{\text {tot }}(\mathrm{M} 07) \\
\left(\mathrm{cm}^{-2}\right)\end{array}$ \\
\hline \multicolumn{4}{|c|}{ System A $z_{\text {abs }}=2.44$} \\
\hline H I & $15.124 \pm 0.030$ & & \\
\hline CIV & $15.270 \pm 0.105$ & 15.236 & \\
\hline $\mathrm{NV}$ & $14.527 \pm 0.102$ & & \\
\hline Si IV & $12.250 \pm 0.164$ & & \\
\hline Si III & (a) & & \\
\hline \multicolumn{4}{|c|}{ System B $z_{\mathrm{abs}}=2.48$} \\
\hline H I & $15.950 \pm 0.713$ & & \\
\hline CIV & $15.471 \pm 0.085$ & $15.024 \pm 0.047$ & $15.144 \pm 0.162$ \\
\hline C II & $14.956 \pm 0.025$ & $14.905 \pm 0.151$ & $15.570 \pm 0.997$ \\
\hline $\mathrm{NV}$ & $14.085 \pm 0.151$ & $13.663 \pm 0.275$ & $13.730 \pm 0.023$ \\
\hline O I & $13.965 \pm 0.116$ & $14.390 \pm 0.032$ & $14.608 \pm 0.028$ \\
\hline Mg II & $15.106 \pm 0.155$ & & \\
\hline (i) & $13.493 \pm 0.062$ & & \\
\hline $\operatorname{Mg} I$ & $11.766 \pm 0.209$ & & \\
\hline $\mathrm{Al} \mathrm{III}$ & $13.260 \pm 0.085$ & & \\
\hline $\mathrm{Al} \mathrm{II}$ & $14.522 \pm 0.237$ & $12.964 \pm 0.023$ & $13.165 \pm 0.889$ \\
\hline & $12.542 \pm 0.113$ & & \\
\hline Si IV(2a) & $14.037 \pm 0.032$ & $13.909 \pm 0.020$ & $14.017 \pm 0.014$ \\
\hline Si III & $16.693 \pm 0.279$ & $14.880 \pm 0.470$ & $15.328 \pm 0.301$ \\
\hline (iii) & $15.902 \pm 0.056$ & & \\
\hline $\operatorname{Si~II(3)~}$ & $14.298 \pm 0.084$ & $15.977 \pm 0.471$ & $15.783 \pm 0.354$ \\
\hline $\mathrm{Fe}$ III & & & \\
\hline $\mathrm{Fe} \mathrm{II}(4)$ & $13.449 \pm 0.064$ & $13.600 \pm 0.030$ & $13.650 \pm 0.010$ \\
\hline \multicolumn{4}{|c|}{ System C $z_{\text {abs }}=2.54$} \\
\hline H I & $15.659 \pm 0.270$ & & \\
\hline C IV & $13.510 \pm 0.155$ & $13.440 \pm 0.110$ & $13.410 \pm 0.050$ \\
\hline \multicolumn{4}{|c|}{ System D $z_{\text {abs }}=2.55$} \\
\hline H I & $16.081 \pm 1.756$ & & \\
\hline C IV & $15.272 \pm 0.967$ & $14.919 \pm 0.200$ & $16.662 \pm 0.060$ \\
\hline $\mathrm{N} \mathrm{V}$ & $13.191 \pm 0.105$ & & \\
\hline Si IV(2a) & $13.665 \pm 0.072$ & $13.514 \pm 0.091$ & $13.567 \pm 0.023$ \\
\hline \multicolumn{4}{|c|}{ System E $z_{\text {abs }}=1.89$} \\
\hline CIV & $14.050 \pm 0.024$ & $14.054 \pm 0.163$ & $14.034 \pm 0.074$ \\
\hline SiIV \# & $13.036 \pm 0.064$ & & \\
\hline \multicolumn{4}{|c|}{ System J (new system) $z_{\text {abs }}=1.93$} \\
\hline $\mathrm{CIV} \ddagger$ & $13.727 \pm 0.081$ & & \\
\hline \multicolumn{4}{|c|}{ System F $z_{\text {abs }}=1.97$} \\
\hline CiV & $14.618 \pm 0.072$ & $15.840 \pm 3.410$ & $14.505 \pm 0.024$ \\
\hline \multicolumn{4}{|c|}{ System G $z_{\text {abs }}=2.07$} \\
\hline CIV & $14.740 \pm 0.410$ & $14.227 \pm 0.054$ & $14.331 \pm 0.021$ \\
\hline Si IV(2b) & $13.587 \pm 0.162$ & $13.260 \pm 0.054$ & $13.327 \pm 0.017$ \\
\hline \multicolumn{4}{|c|}{ System I $z_{\mathrm{abs}}=2.18$} \\
\hline CIV & $13.928 \pm 0.023$ & & $14.019 \pm 0.072$ \\
\hline Si IV & $12.991 \pm 0.032$ & & $12.797 \pm 0.015$ \\
\hline Si III & & & $12.800 \pm 0.022$ \\
\hline \multicolumn{4}{|c|}{ System K (new system) $z_{\mathrm{abs}}=2.26$} \\
\hline H I & $14.850 \pm 0.615$ & & \\
\hline C IV & $12.827 \pm 0.332$ & & \\
\hline \multicolumn{4}{|c|}{ System H $z_{\text {abs }}=2.27$} \\
\hline $\mathrm{HI}$ & $14.074 \pm 0.570$ & & \\
\hline CiV & $13.200 \pm 0.035$ & $13.230 \pm 0.330$ & $13.914 \pm 0.141$ \\
\hline Si IV & $12.527 \pm 0.053$ & & \\
\hline Si III & $12.784 \pm 0.085$ & $13.020 \pm 2.410$ & $12.807 \pm 0.119$ \\
\hline
\end{tabular}

M05 data taken from their Tables 1 and 2 (second spectrum in both cases). M07 data taken from their Table 3. The symbols denote: (i) - without blended line 4.Mg II in system B; (ii) - without blended line 4.Al II in system B; (iii) - without blended line 2.Si III in system B; (a) - but ion detected, but $N$ measurements are unreliable due to blends; (b) - Fe III in System B are detected in the MMT spectrum, but noise is too strong in order to fit this line in the Keck spectrum. $\sharp$ - heavy-element line in Ly $\alpha$ forest region of the spectrum; $\neq-$ blend.

\subsubsection{System $B, z_{a b s}=2.48$}

Even the medium resolution spectrum from D99 clearly showed that this is a strong and complex system. The Ly $\alpha$ absorption was very broad, and its non-symmetric profile hinted that it is in fact a blend of at least a few lines.

The Subaru spectrum allowed this complex to be resolved into 18-19 separate components. As mentioned earlier, our spectra could be satisfactorily fitted with only eight clouds. We show 


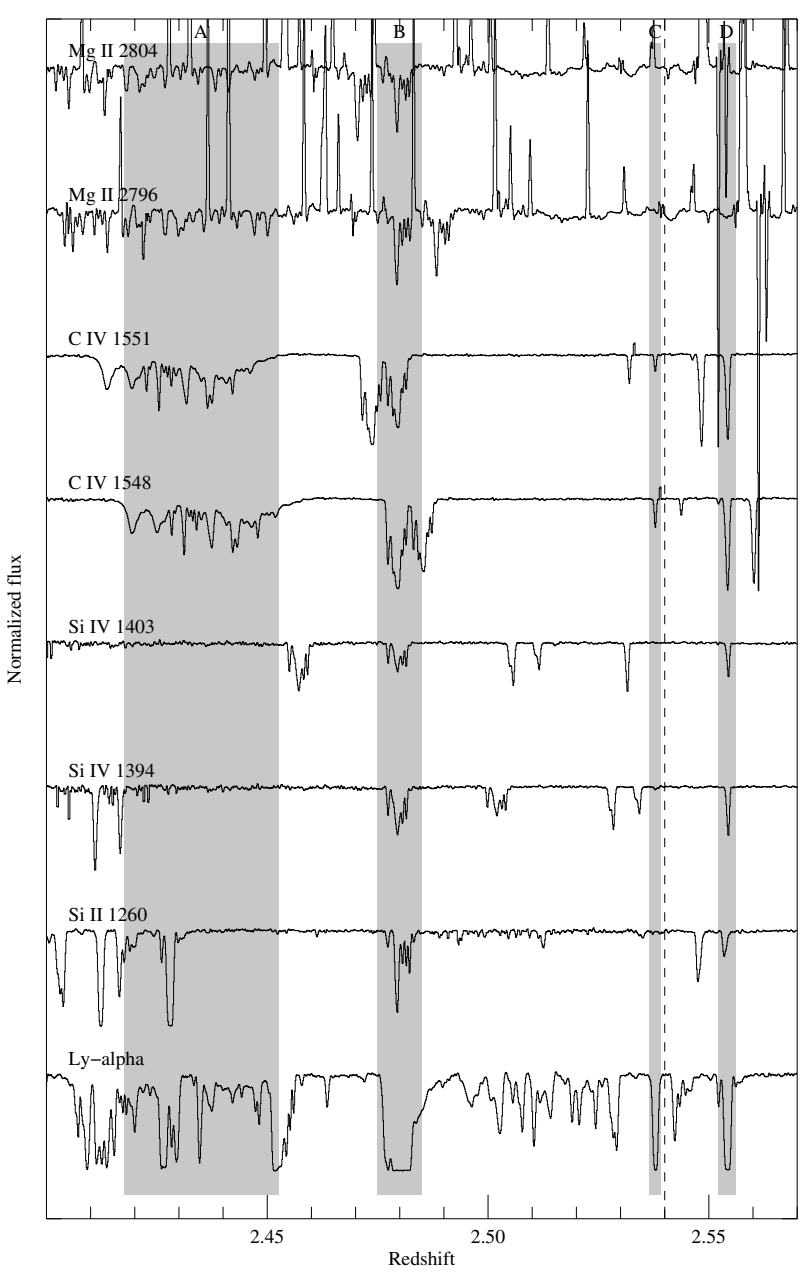

Fig. 2. The fragments of the Keck spectrum of HS1603+3820 plotted in the same redshift range for several absorption features. Systems A-D are shown as shaded areas. The Mg II lines are near the very red end of the spectrum, and the "emission" features seen in their vicinity are residuals of sky lines. The dotted vertical line shows the adopted location of the QSO emission redshift.
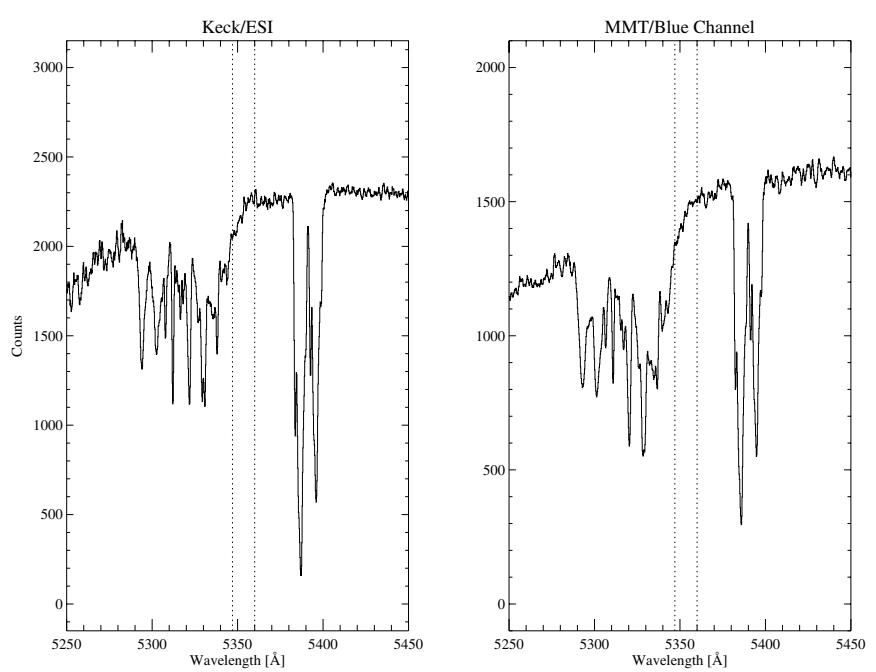

Fig. 3. The sample raw spectra from Keck (left panel) and MMT (right panel) as extracted from the echelle order, prior to continuum fitting. The dotted lines show the $\sim 5347-5360 \AA$ A region where our continuum fit differs from Misawa et al. See text.
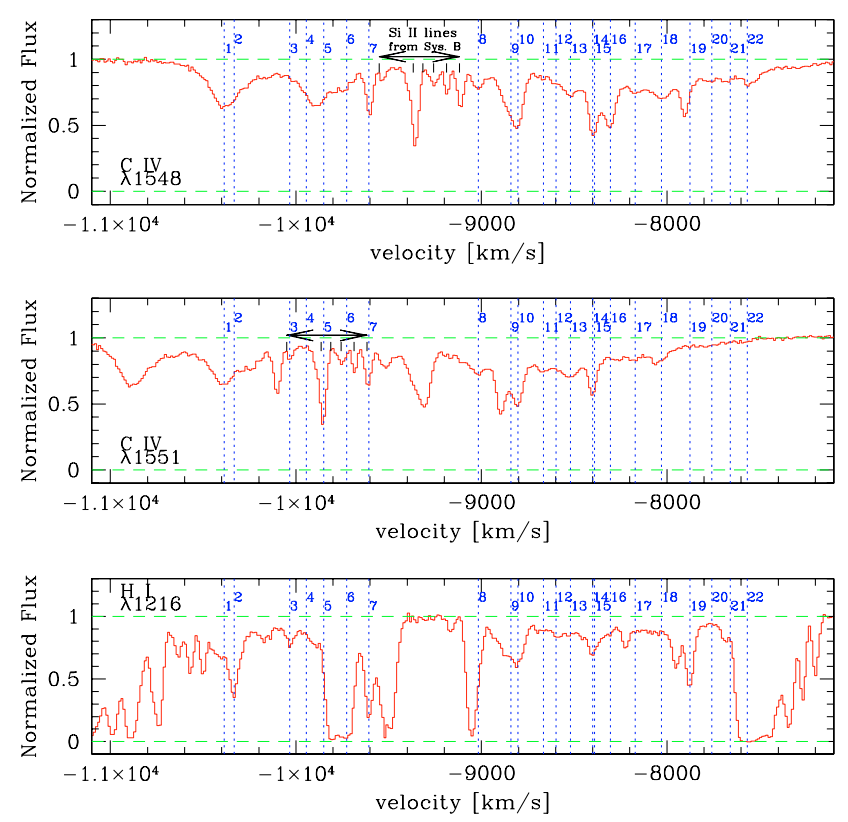

Fig. 4. Absorption lines from System A. Top panel: C IV( $\lambda 1548)$, middle panel: $\mathrm{C} \operatorname{IV}(\lambda 1551)$, bottom panel: Ly $\alpha$. Vertical dotted lines with numbers show the positions of individual lines as listed in Table 1.

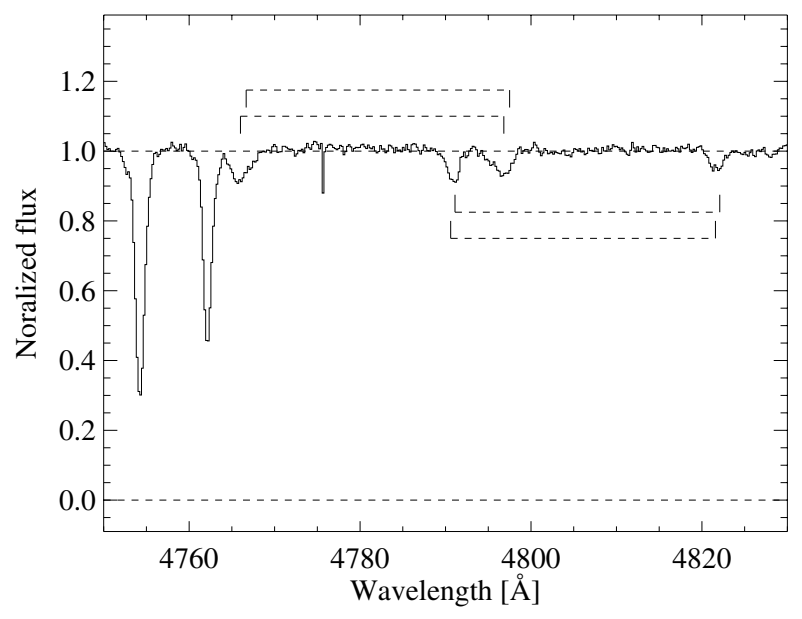

Fig. 5. The Si IV $\lambda \lambda 1394,1403$ doublets from System A.

the vicinity of Ly $\alpha$ and C IV lines in Fig. 6. The derived properties of all lines are listed in Table 2. Note that this system contains lines from as many as fourteen distinct ions.

It needs to be noted, however, that in a few cases the line properties are uncertain. In particular, the seemingly large column densities of some of the $\operatorname{Si} \operatorname{III}(\lambda 1206)$ lines (Nos. 2 and 6 in Table 2) very likely originate from blending with the hydrogen lines. Several other components are also likely affected by features in the spectra; some of those features may be unidentified lines, while some are very likely to be spectral artifacts. All those cases are marked in Table 2.

Also, direct fit of Si II (7.Si II in Table 2) and 4.Fe II in the Keck spectrum resulted in unrealistically high column densities. This was confirmed by visual inspection. The only workaround was to perform the fit to those lines by trial-and-error, then fix the 

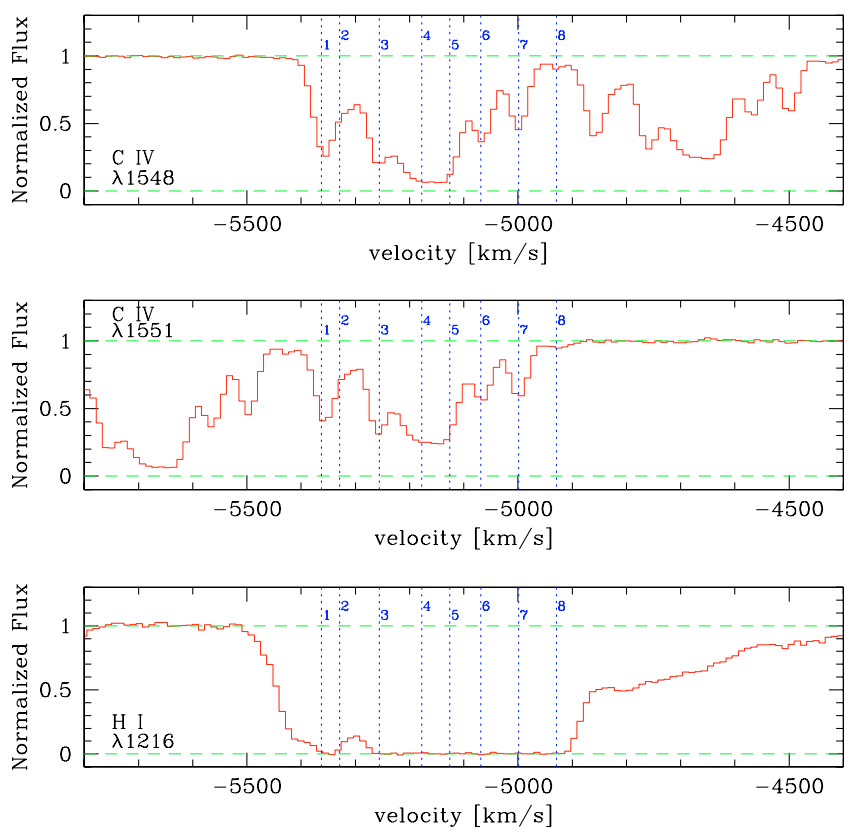

Fig. 6. System B: the C IV (upper panel) and Ly $\alpha$ (lower panel) regions. Positions of lines from Table 2 are marked.

column density for those components, and then refit the whole spectrum again.

The 4.O I lines near $\sim 4531 \AA$ are affected by the C IV doublet from the J system (see below).

The reality of the 5.Al III, 5.Al II and 5.Fe II lines is somewhat uncertain. The programme fitted the lines and they were seen in visual inspection of the spectrum, and we decided to keep them in Table 2. However, we note that the lines themselves and their vicinity are rather noisy.

Total ion column densities seen in our spectra agree well with those seen in the Subaru data (Table 5). Differences can be seen for some ions, but they can be resolved when blends and suspected blends are taken into account.

\subsubsection{System $C, z_{a b s}=2.54$}

This system (see Fig. 7) is very close to the emission redshift of the quasar. In D99 it was considered to be in-falling because of the QSO emission redshift of 2.51 adopted in this paper. However, the arguments presented in M03 favoring a higher value of the emission redshift, $z_{\mathrm{em}}=2.54$, are convincing and we adopt this value in both this paper and in Paper II.

The derived properties of C IV absorption (see Table 3) in this system agree well with the Subaru data. In our data we also see the Ly $\alpha$ line. There is no significant absorption seen in other lines, including Mg II.

\subsubsection{System $D, z_{a b s}=2.55$}

This system is undoubtedly a $z_{\mathrm{abs}}>z_{\mathrm{em}}$ system (see Fig. 8), even with the QSO emission redshift of 2.54. The natural explanation is that this system is infalling onto the quasar.

Misawa et al. resolved this system into four separate components, and we see the very same absorbers in our data. For all of them we identify Ly $\alpha$ and C IV lines.

The stronger two components (lines Nos. 3 and 4 in Fig. 8 and Table 3 ) also show Si IV and N v lines. We list N v only under component No. 4, but it is very likely a blend of components
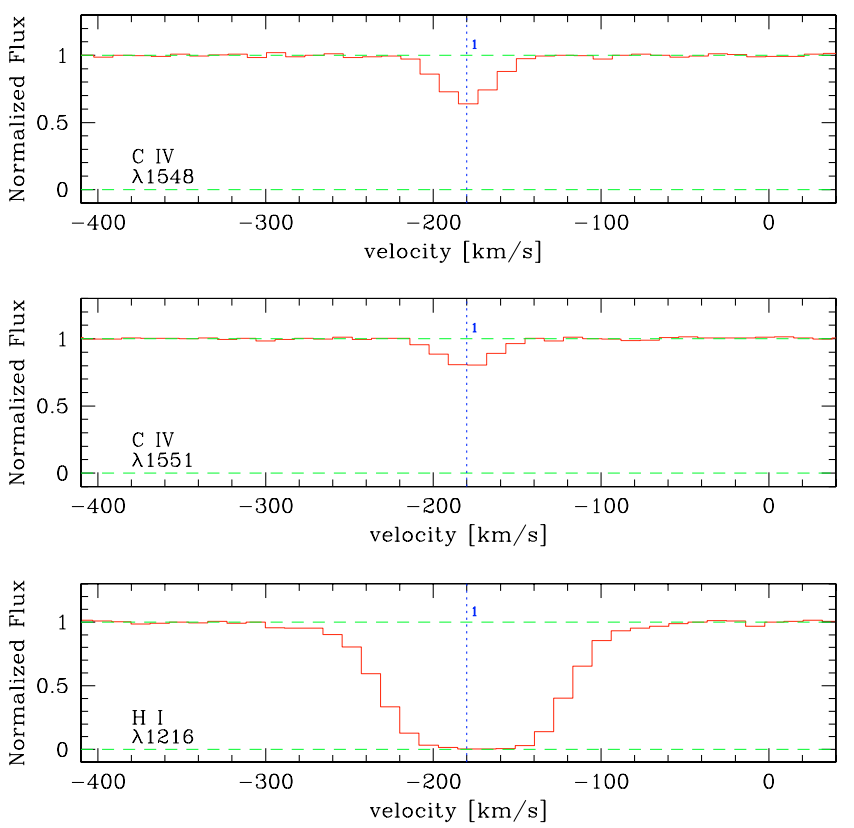

Fig. 7. The CIV (upper panel) and $\operatorname{Ly} \alpha$ (lower panel) regions of System C. Positions of lines detected in the spectra are shown with dotted lines and labeled with the IDs from Table 3.
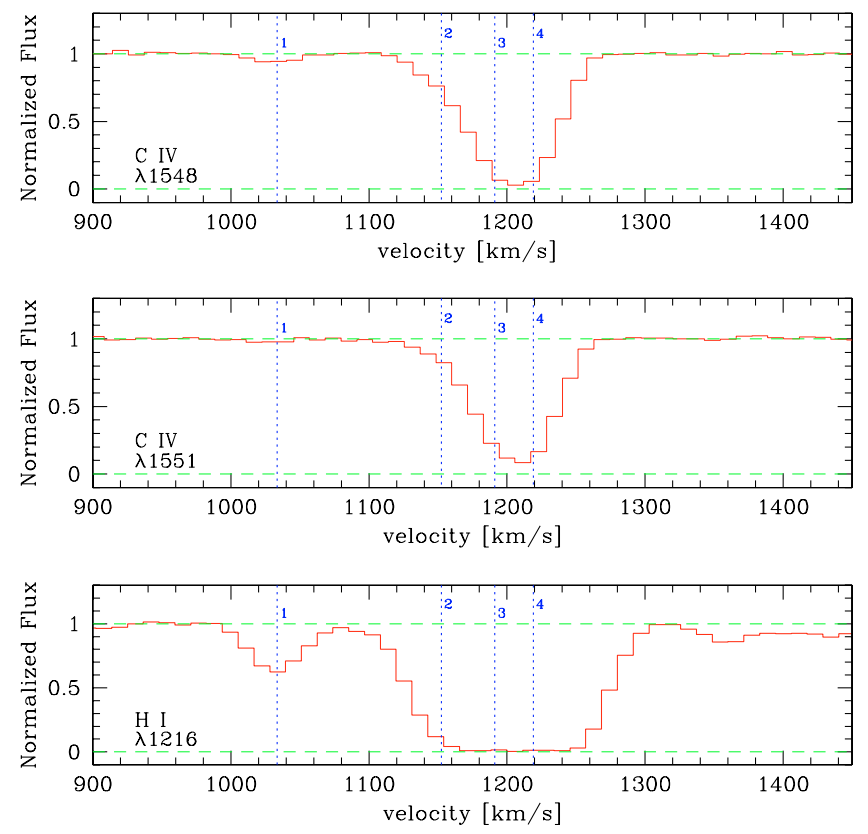

Fig. 8. As in Fig. 7, for System D.

Nos. 3 and 4, since both lines in the doublet are broader than what would be expected based on the value of the Doppler parameter of the other lines. The nitrogen absorption is certainly real, but the troughs are shallow and are only visible because of high signal-to-noise of our spectra; resolving it into two components would be meaningless.

In the case of the Keck spectrum, the fits of the Ly $\alpha$ lines Nos. 3 and 4 were diverging when they were combined with lines from other ions. We therefore fitted them separately and then fixed the values; this is reflected in Table 3, where their values of $N$ and $b$ are listed as fixed.

We also note that the Hi line No. 1 in the Keck spectrum shows an unusually low value of the Doppler parameter. 
However, this was confirmed by the visual inspection of the spectrum: the line is isolated but does appear to be very narrow. Since the corresponding C IV absorption is unambiguously identified, we have no doubts about the reality of this line, and we attribute the unusually low measured value of $b$ to noise fluke.

\subsection{Systems with $Z_{\mathrm{abs}} \ll Z_{\mathrm{em}}$}

The spectrum of HS1603+3820 contains several other heavyelement systems at $z_{\mathrm{abs}} \ll z_{\mathrm{em}}$. We identified seven of them in our spectra; the lists of lines are shown in Table 4. Four of those systems, $\mathrm{E}\left(z_{\mathrm{abs}}=1.89\right), \mathrm{F}\left(z_{\mathrm{abs}}=1.97\right), \mathrm{G}\left(z_{\mathrm{abs}}=2.07\right)$, and $\mathrm{H}$ $\left(z_{\text {abs }}=2.27\right)$, were identified in the early Subaru spectra, while System I $\left(z_{\text {abs }}=2.18\right)$ was described in the recent paper by M07.

In almost all cases, the properties derived from our spectra match reasonably well with those from the Subaru data. The two exceptions are the Nos. 3 and 4 C IV lines from System F. In the first case M03 and M05 have a doublet of lines of similar strength separated by $\sim 7 \mathrm{~km} \mathrm{~s}^{-1}$, which in our spectrum is not deblended, and the second case is a weak line buried in a nearby, stronger line. The total column density derived from our data does, however, agree well with the Subaru value (Table 5).

Although the Si IV $(\lambda \lambda 1394,1403)$ lines in System E (Fig. 9) lie in the Ly $\alpha$ forest, they are clearly identified in both Keck and MMT spectra. Existence of the second Si IV doublet is rather uncertain. We could not see it in the Keck data, although it is perhaps present in the MMT spectrum.

The natural interpretation for the origin of the systems with $z_{\mathrm{abs}} \ll z_{\mathrm{em}}$ is that they are intervening, i.e., they are caused by objects that happen to be placed on the line of sight to the quasar but are not related to it, although there are cases in the literature where convincing arguments were presented that systems seemingly far from the quasar could be associated (e.g. Hamann et al. 1997; Richards et al. 1999). M05 indicated that Systems E and F could be associated, since their analysis seemed to show that some of the components in those systems had partial coverage, although the lines did not show any variability. In our analysis we obtained satisfactory profile fits without the assumption of partial coverage. This accounts for the difference between line parameters derived here and in the Subaru spectra. However, the total column densities agree well (Table 5).

In our spectra, we also identify two systems that are not seen in the Subaru spectra. We label them J $\left(z_{\mathrm{abs}}=1.9266\right)$ and $\mathrm{K}$ $\left(z_{\mathrm{abs}}=2.2622\right)$; the line properties are listed in Table 4 . We show the two systems in Fig. 10.

System $\mathbf{J}$ requires a special comment. The Ly $\alpha$ line for this system would be outside of the wavelength region covered by both MMT and Keck spectra, and the only identified feature is the $\mathrm{C}$ IV doublet. Before we proceed, we note that it is somewhat surprising that the redder of the two lines, at $\lambda_{\text {obs }} \approx 4538 \AA$, is apparently not identified in any of the Subaru spectra, since none of the line lists seem to contain it. This line is unambiguously present in both of our spectra.

What makes this system somewhat uncertain is the fact that both those lines coincide very well with the locations of what we expect to be the strongest $\mathrm{O} \mathrm{I}(\lambda 1302)$ and $\mathrm{Si} \mathrm{II}(\lambda 1304)$ lines from System B. However, there are indications that C IV is in fact present in this location. First, the spectra contain several other Si II lines, and they constrain the column density of that ion quite well, suggesting that Si II( 21304$)$ alone cannot account for all of the absorption in the redder of the two lines. Second, for O I to be responsible for all of the absorption in the bluer of the two

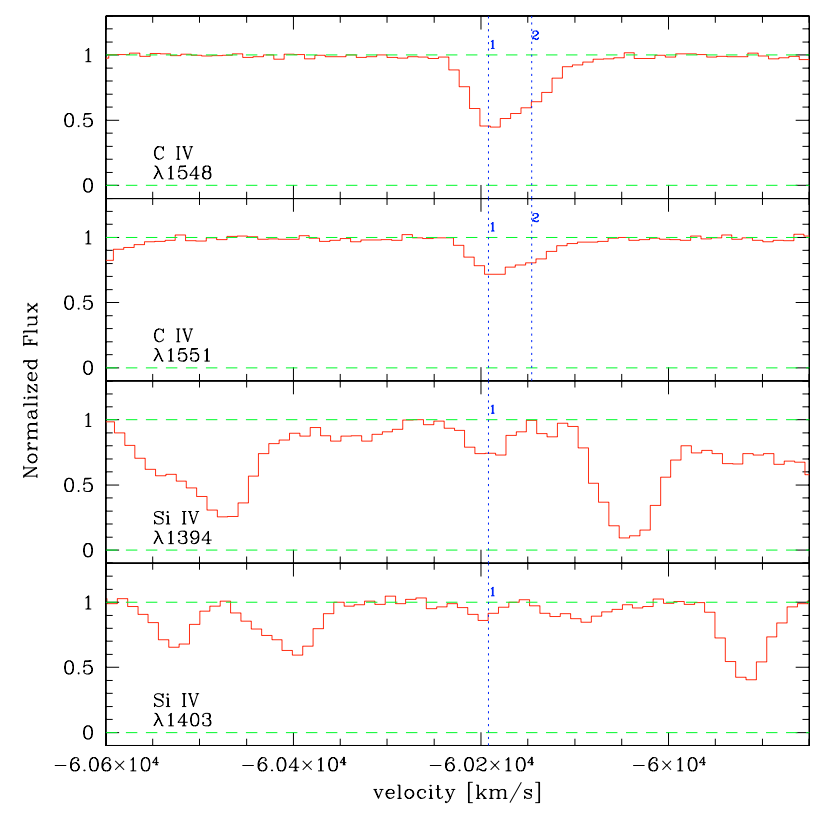

Fig. 9. The CIV (upper panel) and Si IV (lower panel) regions for System E. Markings as in Fig. 7.
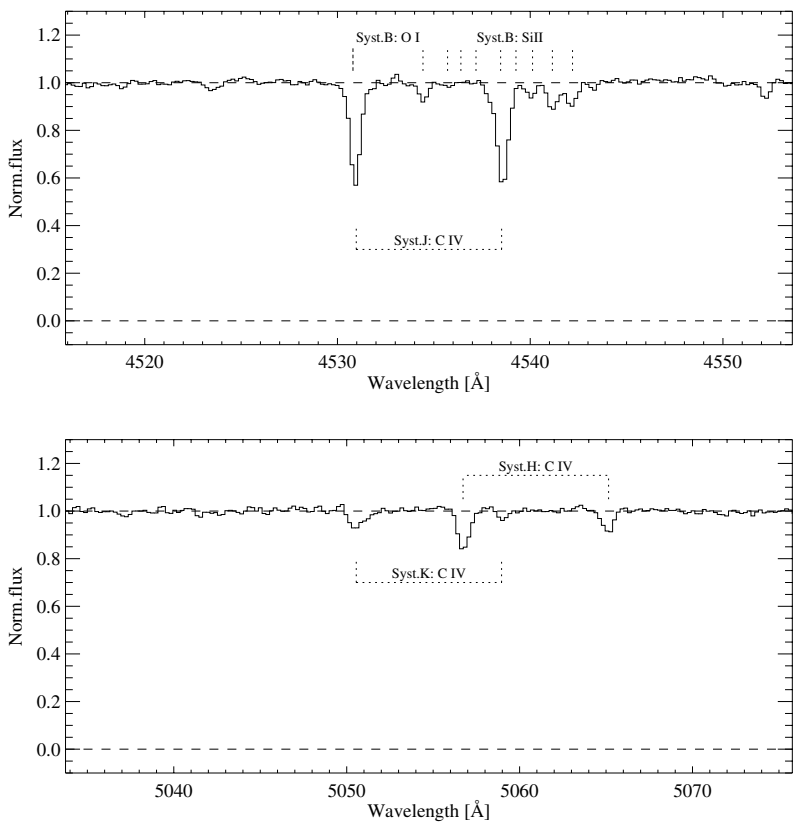

Fig. 10. The C IV regions for System J (upper panel) and System K (lower panel).

lines, at $\lambda_{\mathrm{obs}} \approx 4530 \AA$, it would require an O I column density of $\sim 10^{15} \mathrm{~cm}^{-2}$, which we consider implausible.

The above considerations lead us to believe that System $\mathrm{J}$ is real, although the fact that both lines are blends makes its properties rather uncertain.

Another of the previously unidentified systems is a system at $z_{\text {abs }}=2.2622$, which we label K. It is clearly seen in both our spectra; obviously, we could identify it because of the high signal-to-noide ratio in our data. The system is displaced by $\sim 400 \mathrm{~km} \mathrm{~s}^{-1}$ from System H. It is thus likely that both these systems originate in objects from the same group of galaxies. 


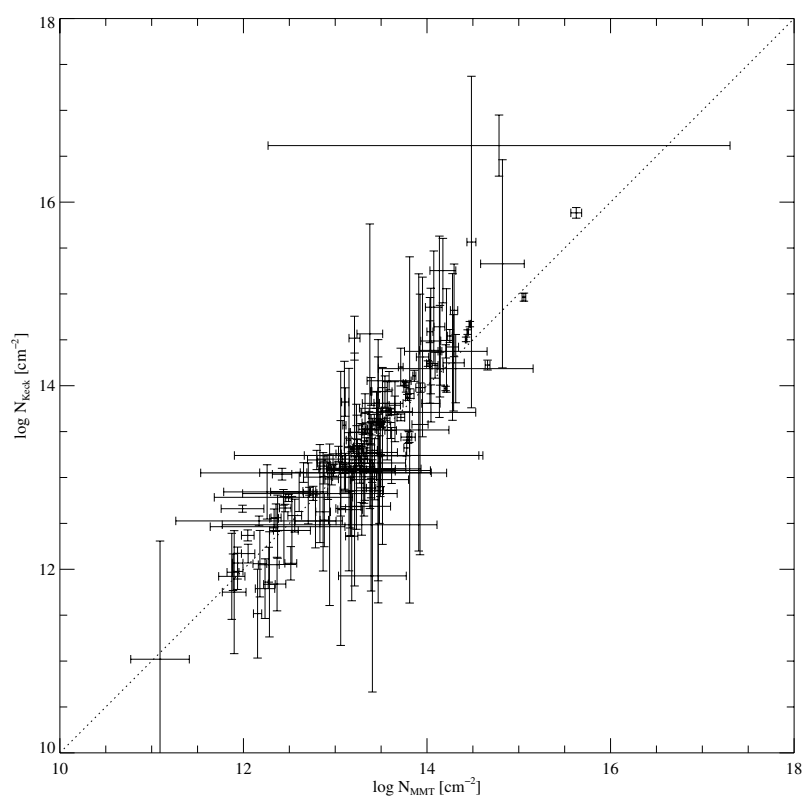

Fig. 11. Comparison of column densities of all lines for which measurements exist from both MMT ( $x$-axis) and Keck ( $y$-axis) data. See text for discussion.

\section{Notes on the variability of the absorbers}

Misawa et al. present convincing arguments that the System A absorber is variable, but they did not see variability in any of the other systems. In general, unlike the Subaru observations, our optical data are not well suited for the analysis of absorber variability. First, we have data from two epochs only. Second, the data were obtained with two different telescopes/instruments, at different locations and under different atmospheric conditions not to mention that one of the observations was likely affected by smoke from forest fires. Third, one of the extracted spectra had issues with residual flux. Fourth, in some places the continuum fits had to be adjusted manually. Fifth, the variability range reported in M07 was of the order of $\Delta \log N \sim 0.3$, which is only slightly higher than our typical measurement uncertainties. All these factors make direct comparison of the derived properties of the absorption lines questionable, and especially in the regions affected by the continuum fit uncertainty. Any difference between absorber properties derived from the two spectra will likely contain an unknown component attributable to these issues.

Being aware of all these considerations, we examined the column densities derived from the MMT and Keck data looking for any systematic differences between the two datasets. In Fig. 11 we show column densities for the same components as derived from the MMT and Keck data. Note that the error bars only show formal uncertainties as derived from the fits and do not include the unknown uncertainty related to the analysis issues. Despite this fact, one can clearly see that the properties of components as derived from our two observations agree very well. Only very few data points deviate from the $N_{\text {Keck }}=N_{\text {MMT }}$ line by more than $1 \sigma$, and the ones that do are practically all in the high- $N$ region, where issues related to the residual flux in the MMT spectrum are expected to play a role.

This conclusion applies to both $z_{\mathrm{abs}} \approx z_{\mathrm{em}}-$ including System A, which Misawa et al. have shown to be variable - as well as the $z_{\mathrm{abs}} \ll z_{\mathrm{em}}$ systems. For the latter systems this is not surprising, although, as mentioned earlier, there were claims in the literature (e.g. Hamann et al. 1997; Richards et al. 1999) indicating that systems with large redshift difference show variability (see also Hao et al. 2007).

\section{Summary}

The following summarizes our findings:

- The intrinsic X-ray properties of HS1603+3820 are in the typical range. The available X-ray data were insufficient to provide meaningful constraints on the properties of the intrinsic absorber. The optical-to-X-ray slope, $\alpha_{\mathrm{ox}}=1.70$, is at the high end of the typical range at this redshift.

- In addition to systems seen in D99 and Misawa et al.'s papers, we identify two previously unknown intervening systems at $z_{\mathrm{abs}}=1.9266$, and 2.2622. With eleven complexes of heavy element absorbers, which together contain 50 (or even more) individual clouds - and that is not counting Ly $\alpha$ forest systems - HS1603+3820 is truly a spectacular object.

- Some of the clouds in System A have a high ratio of C IV to H I column densities, reaching $\sim 20$. Clouds from other systems show $N_{\text {CIV }} / N_{\text {H I }}<1$.

- The data presented in this paper are not well suited for proper analysis of line temporal variability. We note, however, that the data are consistent with absence of variability for all systems.

- The optical data presented in this paper contain identifications and measurements of several lines not seen in the Subaru spectra, in particular, the hydrogen and magnesium lines.

- Our data contain measurements of column densities of several ions and therefore provide excellent constraints for modeling of the cloud internal properties and QSO intrinsic flux. We will present such modeling in the forthcoming paper.

Acknowledgements. We thank J. Liske for his help with the VPGUESS/VPFIT packages, T. Aldcroft and A. Siemiginowska for helpful comments, and the referee for a very careful review. M.N. would like to thank ESO for hospitality during his stay there. This work was partially supported by the ESO Director General Discretionary Fund, by the National Aeronautics and Space Administration through Chandra Award Number 04700527 issued by the Chandra X-ray Observatory Center, which is operated by the Smithsonian Astrophysical Observatory for and on behalf of the National Aeronautics Space Administration under contract NAS8-03060, and by grant 1P03D00829 of the Polish State Committee for Scientific Research.

\section{References}

Aldcroft, T. L., Bechtold, J., \& Elvis, M. 1994, ApJS, 93, 1

Bechtold, J., Siemiginowska, A., Shields, J., et al. 2003, ApJ, 588, 119 Crenshaw, D. M., Kraemer, S. B., Gabel, J. R., et al. 2003, ApJ, 594, 116 Dobrzycki, A., Engels, D., Hagen, H.-J., et al. 1996, BAAS, 28, 829 Dobrzycki, A., Engels, D., \& Hagen, H.-J. 1999, A\&A, 349, L29

Fiore, F., Elvis, M., Giommi, P., \& Padovani, P. 1998, ApJ, 492, 79 Gabel, J. R., Kraemer, S. B., Crenshaw, D. M., et al. 2005, ApJ, 631, 741 Hamann, F., Barlow, T. A., Junkkarinen, V., \& Burbidge, E. M. 1997, ApJ, 478, 80

Hao, H., Stanek, K. Z., Dobrzycki, A., et al. 2007, ApJ, 659, L99

Misawa, T., Yamada, T., Takada-Hidai, M., et al. 2003, AJ, 125, 1336

Misawa, T., Eracleous, M., Charlton, J. C., \& Tajitsu, A. 2005, ApJ, 629, 115

Misawa, T., Eracleous, M., Charlton, J. C., \& Kashikawa, N. 2007, ApJ, 660, 152

Reeves, J. N., \& Turner, M. J. L. 2000, MNRAS, 316, 234

Richards, G. T., York, D. G., Yanny, B., et al. 1999, ApJ, 513, 576

Scott, J., Bechtold, J., Dobrzycki, A., \& Kulkarni, V. P. 2000, ApJS, 130, 67

Scott, J. E., Kriss, G. A., Lee, J. C., et al. 2005, ApJ, 634, 193

Sheinis, A. I., Bolte, M., Epps, H. W., et al. 2002, PASP, 114, 851

Véron-Cetty, M. P., \& Véron, P. 2006, VizieR Online Data Catalog, 7248 